\title{
Involvement of FLOWERING LOCUS T in microgravity response of Arabidopsis thaliana plants under long- and short-day conditions
}

\section{Lihua Wang}

Chinese Academy of Sciences

Junyan Xie

Chinese Academy of Sciences

Yuanyuan Wu

Chinese Academy of Sciences

Chenghong Mou

Chinese Academy of Sciences

Yuwei Jiao

Chinese Academy of Sciences

Yanhui Dou

Chinese Academy of Sciences

Huiqiong Zheng ( $\nabla$ hqzheng@cemps.ac.cn )

Chinese Academy of Sciences

\section{Research Article}

Keywords: Photoperiod, Microgravity, F lowering, HSP17.4, FLOWERING LOCUS T, Transcriptome

Posted Date: April 15th, 2021

DOI: https://doi.org/10.21203/rs.3.rs-400169/v1

License: (c) (1) This work is licensed under a Creative Commons Attribution 4.0 International License.

Read Full License 
Involvement of FLOWERING LOCUS T in microgravity response of Arabidopsis thaliana plants under long- and short-day conditions

Lihua Wang $\dagger^{1}$, Junyan Xie $\dagger^{1}$, Yuanyuan $\mathrm{Wu}^{1,2}$, Chenghong $\mathrm{Mou}^{1,2}$, Yuwei Jiao ${ }^{1,2}$, Yanhui Dou ${ }^{1,2}$, Huiqiong Zheng ${ }^{1 *}$

${ }^{1}$ CAS Center for Excellence in Molecular Plant Sciences, Chinese Academy of Sciences, Shanghai, 200032, China

${ }^{2}$ University of Chinese Academy of Sciences, Beijing 100049, China

*Correspondence author

Phone: 86-21-54924243

Fax: 86-21-54924015

Email: hqzheng@cemps.ac.cn

$\uparrow$ The authors have contributed equally.

Number of words in main text: 4341;

Number of figures: 9

Number of tables: 2

Number of supplementary tables: 11 


\begin{abstract}
Microgravity have an impact on growth and development of higher plants in space at both vegetative stage and reproductive stage. A great deal of information has been available on the vegetative stage in space, but relatively little is known about the influence of microgravity on plants at the reproductive stage. In this study, we constructed a transgenic Arabidopsis thaliana plants expressing flowering control gene, FLOWERING LOCUS T (FT), together with green fluorescent protein gene $(G F P)$ under control of a heat shock-inducible promoter (HSP17.4), by which we induced $F T$ expression inflight through remote controlling heating shock treatment. Inflight photography data showed that induction of FT expression in plants in space could counteract the impact of microgravity and promote flowering. Whole-genome microarray analysis of gene expression changes in leaves of wild-type and these transgenic plants grown under different photoperiod conditions in space indicated that the function of the photoperiod-related microgravity response genes are mainly involved in protein synthesis and post-translation protein modulation, notably protein phosphorylation. In addition, changes of circadian component gene expression in response to microgravity under different photoperiod indicated that role of circadian oscillator could act as integrators of microgravity response and photoperiodic signals in Arabidopsis plant grown in space.
\end{abstract}

Key words: Photoperiod; Microgravity; Flowering; HSP17.4; FLOWERING LOCUS T; Transcriptome. 
2 Microgravity by spaceflight could cause an impact on growth and development of

3 higher plants at both the vegetative stage and the reproductive stage. A great deal of

4 information is available on the vegetative stage in space. For example, alteration of

5 auxin polar transport in etiolated pea seedlings and maize coleoptiles in space (Ueda

6 et al., 2000), inhibition of cell division and mitosis as well as significant

7 karyological disturbances in root-tip cells of oat, mung bean and sunflower

8 seedlings grown in space, modification of cell wall metabolism (Krikorian and

9 O’Conner, 1984; Rasmussen et al., 1994, Sago et al., 2002). Reduction in fresh

10 weight of shoot and photosynthetic function of wheat plants grown onboard space

11 shuttle (Tripathy et al., 1996). The plants grown in space was often smaller than

12 comparably aged ground controls (Kiss et al., 2000; Paul et al., 2012; Wang et al.,

13 2018), while others grew faster in space (Matía et al., 2010; Hoson et al., 2014).

14 However, relatively little is known about the influence of microgravity on plants at

15 the reproductive stage. Some early experiments reported failure in seed formation

16 under spaceflight conditions (Nechitatio and Maskinsky, 1993; Kuang et al., 1996;

17 Strickland et al., 1997; Soga et al., 1999; Levinskikh et al, 2000; Campbell et al., 18 2001). As hardware improvement, the completion of the seed-to-seed cycle of 19 several plants in space were reported (Stankovic, 2001; Link et al., 2003; Sychev et 20 al., 2003; Link et al., 2014). These results indicate that plants could adapt to 21 microgravity for seed-to-seed growth, but reproductive fitness is often reduced in 22 space (De Micco, et al., 2014). Interruption of the reproductive process, delay in completion of single reproductive phase, lowering of reproductive success and

24 alteration of seed reserves are still major bottlenecks to maximize the efficiency of 25 plant growth and reproduction in space and to be used to support life in long-term 
manned missions (Hoson, 2014; reviewed by Zheng 2018).

27 The reproductive success of plants is often dependent on their flowering time being 28 adapted to the growth environment. A number of studies suggest that both biotic and 29 abiotic stress factors play key roles in controlling to alter flowering time in plants.

30 For example, plants often acceleration the flowering process under drought stress 31 (Sherrard and Maherali, 2006;Galbiati et al., 2016) and delays flowering time by salt stress (Achard et al., 2006; Ma et al., 2015). Heat and cold stress can also have a dramatic effect on flowering. In addition, the other stresses, such as, nutrient, sugar 34 budget, geomagnetic field and simulated microgravity, have significant effects on plant development including flowering process time (Lee et al., 2008; Posé et al., 2013; Agliassa et al., 2018; Xie et al., 2020). Increasing evidences document that microgravity is a novel stress for plants grown in space (Paul et al., 2001; De Micco et al., 2014; Zhang et al., 2015; Karahara et al., 2020), which cause changes at the physiological, morphological and molecular levels, including altered transcription

40 patterns of many genes. In the space-grown Mizunna, a total of 20 in 32 ROS oxidative maker genes were up-regulated, including common genes response to abiotic and biotic stress (Sugimoto et al., 2014). In Arabidopsis culture cells grown in space, genes associated with heat shock, salt, drought, metals, wounding, 44 phosphate, ethylene, senescence, terpenoids, seed development, cell walls, 45 photosythesis, and auxin were up-regulated by five fold in comparison with their ground controls (Paul et al., 2012; Kwon et al., 2015). The endogenous systems that measure day length was found to interact with stress responses and override interpretation of the signals in plants on ground (Becker et al., 2005). It is however unclear how the photoperiod influence the signals in plants in space. 
51 daylength, because Arabidopsis is a long-day (LD) plant, an increase in photoperiod

52 results in an increase in development rate. How photoperiod affect plant

53 development in space has yet known. No space experiments had been carried out to

54 compare the effects of different photoperiod on plant growth and development so far.

55 To exmamine effects of photoperiod signals on the microgravity response of plants

56 in space, we conducted the space experiment by growing Arabidopsis plants under

57 the LD and the short-day (SD), respectively, on board the Chinese recoverable

58 satellite SJ-10. A transgenic plants expressing FLOWERING LOCUS T (FT) and the

59 reporter gene green fluorescent protein (GFP) under control of a heat

60 shock-inducible promoter (HSP17.4) was constructed to invesitigate the role of FT

61 in integation of microgravity into photoperiod controlling floral pathway. In

62 addition, a full-genome analysis of RNA derived from the leaves of Arabidopsis

63 plants in space under the $\mathrm{LD}$ and the $\mathrm{SD}$, respectively, were also performed in

64 comparison with their controls on ground.

\section{Results}

66 Gene switch for flowering induction in space experiment

67 To address the effects of microgravity on the FT regulating flowering pathway, we

68 generated transgenic Arabidopsis plants that stably harbor FT gene and GFP gene,

69 under control of the HSP17.4 promoter, which have been utilized to establish a

70 highly efficient regulatory system in plants through heat-shock treatment

71 (Czarnecka et al., 1990). Under the LD condition, this $p H S P:: \underline{F T}, p H S P: \underline{G F P}$ (FG)

72 transgenic plants grown on ground at normal temperature $\left(22 \pm 2^{\circ} \mathrm{C}\right)$ exhibit a

73 phenotype like wild-type (WT), except the size is slightly smaller (Supplementary

74 Fig. S1 and S2). In the absence of heat shock (HS), no GFP fluorescence in leaves

75 of FG plants was observed (Supplementary Fig. S1C and G), while heating at $37^{\circ} \mathrm{C}$ 
76 for 30 min resulted in a clear induction of GFP expression in the leaves of FG plants

77 (Supplementary Fig. S1D and H), but didn't in the leaves of WT plants

78 (Supplementary Fig. S1B and F). Early floral development and apparent increase of

79 FT gene expression in FG plants under the SD conditions (Supplementary Fig. S2)

80 were also observed after HS induction, while the control plants (WT and

$81 p H S P:: G F P)$ with or without exposure HS treatments showed negligible levels of

82 background FT expression and little GFP fluorescent under the same condition

83 (Supplementary Fig.S1; Supplementary Fig. S2G).

84 For space experiment on the satellite SJ-10, seeds of WT and FG were germinated and grown in the root modules on ground under the LD condition for 20 days (Fig. $1 \mathrm{E}$; corresponding to stage 1.06, Boyes et al., 2001). At this age, the plants had formed about 5-6 rossette leaves (Fig.1E and F), when they were loaded into the plant growth unit (PGU) less than 24h prior to take off. Under the LD condition,

89 floral shoots of WT plants on ground appeared at day 4 after satellite launched, 90 while plants in space initiated floral shoots on day 6 (Fig. 2A and B). For FG plants, 91 floral shoots appeared at day 2 under the LD on ground were earier than those in 92 space at day 4 (Fig. 2A and C), slightly earier that WT under the same condition. No 93 apparent GFP signal was detected in leaves of WT and FG plants before $37^{\circ} \mathrm{C} \mathrm{HS}$ 94 induction (Fig.3 D and E). A strong transient expression of the transgenic GFP 95 fluorescence in leaves of FG plants in space and on ground were detected under the SD condition after 24h HS-treatment, while no signal appeared in leaves of WT

97 plants under the same conditions (Fig.3H-O ). The highest abundance of GFP signal 98 in the leaves of HS-treated plants grown under SD in space was obseved at day 8 99 (Fig. 3P). Flowering of FG plants under the SD both in space and on ground 100 exhibited 2 3 days earler than that of WT plants (Fig. 2D, E and F). These results 
indicated that the $p H S P:: F T, p H S P: G F P$ system we constructed in this study could mediate an "on/off" situation of $F T$ gene activity in FG plants by HS treatment and could be used as gene switch for flowering induction both in space and on ground.

Identification of differentially expressed genes in response to microgravity in space under different photoperiodic conditions

To identify the molecular basis on FT integrates microgravity in controlling flowering pathway, the global transcriptional effects of microgravity were monitored in leaves using whole-genome Arabidopsis GeneChips (Affymetrix). RNA was extracted from leaves of WT and FG grown in microgravity $(\mu g)$ on spaceflight under the $\mathrm{LD}(\mathrm{LD}, \mu \mathrm{g})$ and the $\mathrm{SD}(\mathrm{SD}, \mu \mathrm{g})$, and their controls on ground under the $\mathrm{LD}(\mathrm{LD}, 1 \mathrm{~g})$ and the $\mathrm{SD}(\mathrm{SD}, 1 \mathrm{~g})$, respectively. The estimated mean level of gene expression in WT or FG in space were significantly different compared with the estimated mean of controls on ground when controlling the false discovery rate (FDR) at the level of 0.05 using the method of storey and Tibshirani (2003). Of the genes that met these criteria, we rank ordered them by fold change (FC). That expression level changed more than $2(\mathrm{FC} \geq 2)$ were selected as differential expression gene (DEG). The DEGs were comparatively analyzed and divided into five steps (Fig.4 A). Step 1, expression of genes in leaves grown under the $\mathrm{SD}$ on ground was compared with those under the LD on ground (SD, $1 \mathrm{~g}$ vs LD, $1 \mathrm{~g}$, namely SD-1g). This approach allowed us to overveiw the influence of photoperiod on gene expression on ground without alteration of gravity in WT and FG, respectively (Supplementary Table S1 and S2). Step 2, genes altered expression levels in response to microgravity under the LD. For this, gene expression in WT and FG plants grown in space under the LD were compared with those on ground under the LD (LD, $\mu g$ vs $1 g$, namely LD- $\mu g$ ), respectively (Supplementary Table S3 
and S4 ). Step 3, changes of gene expression in response to $\mu g$ occurred in WT and

127 FG plants under the SD condition (SD, $\mu g \nu s 1 g$, namely SD- $\mu g$ ), respectively

128 (Supplementary Table S5 and S6 ). Step 4, Photoperiod related $\mu g$ response genes

129 are selected by comparisons between $\mu g$-LD and $\mu g$-SD response genes in WT and

130 FG plants, respectively(Supplementary Table S7 and S8). Step 5, comparison of

131 altered expression of photoperiod related $\mu \mathrm{g}$ response genes between WT and FG

132 was performed (Supplementary Table S9). For this, "FT related photoperiod

133 controlling $\mu g$ response genes" could be identified.

134 A major impact of microgravity on global transcription

135 Figure 4B showed than 427 genes (7.3\% of total 5863 DEGs) in WT and 477 genes

$136(8.5 \%)$ in FG on ground were altered in transcript abundance under the SD

137 compared to those under the LD on ground (Supplementary Table S1 and S2).

138 Expression of 4432 genes (75.6\%) in WT and 3922 genes (74.5\%) in FG were

139 altered in response to microgravity under the LD (Supplementary Table S3 and S4),

140 while 2571 genes (43.9\%) in WT and 2031genes (38.6\%) in FG changed expression

141 levels by subjected to microgravity under the SD (Supplementary Table S5 and S6).

142 The proportion of genes up-regulated by microgravity was always higher than the

143 down-regulated ones (Fig. 4C). Principal component analysis (PCA) of the samples

144 demonstrated a strong difference between transcriptomes of samples grown on

145 ground and in space under both the LD and the SD (Fig.4D).These results indicated

146 that the number of genes defferential expression in response to microgravity was

147 overall greater than the number of genes controlled by day length, suggesting a

148 major impact of microgravity in space on global transcription in the leaves of WT

149 and FG plants under both LD and SD conditions. 
150 To validate the microarray data, we generated sequence-specific primers and 151 performed real-time RT-PCR on a third independent replicate. Real-time PCR with

152 isoform specific primers for calcium sensing receptor (At5g23060), haloacid

153 dehalogenase-like hydrolase superfamily protein (At3g48420), constans-like

154 2(At3g02380) and TIMELESS (At5g52910) confirmed the relative abundance

155 changes for the transcript levels of these genes of WT and FG in response to

156 microgravity in space under LD and SD conditions (Fig.5).

Daylength related microgravity response genes

158 To characterize the gene categories in response to microgravity under different 159 photoperiod, two groups of genes were divided based on their transcriptional 160 behaviors (Table 1). The genes responding to microgravity under LD and SD conditions with similar behaviors were named ' $\mu$ g-common' genes, whereas those in response to $\mu \mathrm{g}$ specific to $\mathrm{LD}$ or SD were named ' $\mu$ g-daylength-related' genes. Among' $\mu$ g-common 'genes, transcript levels of 1018 genes in WT and 720 genes in FG showed similar changes in response to microgravity under the LD and the SD (Fig. 6A; Table 1; Supplementary Table S7 and S8). In contrast, a relative large number of ' $\mu$ g-daylength-related' genes were found with total 4512 genes in WT and 4201 genes in FG (Table 1; Fig. 6A; Supplementary Table S7 and S8). Overall changes in gene expression pattern in WT and FG for ' $\mu$ g-daylength-related ' genes were apparently more than those of ' $\mu$ g-common', indicating that daylength is an important factor to regulate response of plants to microgravity in space. GO categories representing ' $\mu$ g-common' and ' $\mu$ g- daylength related' genes exhibit

172 similar behaviors in the down-regulation of ribosome biogenesis and RNA processing (i.e. ncRNA, rRNA metabolism and processing), amino acid metabolic

174 process. However, compared with ' $\mu$ g-common' genes, a significantly enriched GO terms were identified for ' $\mu$ g-daylength-related' genes, among which the most overrepresentation was protein phosphorylation GO category in both WT and FG

177 (Fig.6B and C). The function of proteins encoded by these overrepresented 
178 ' $\mu$ g-daylength-related' genes are involved in light and ethylene signaling, calcium

179 signaling, cell wall-associated receptor kinase-like proteins, phosphatase and protein 180 kinase and others (Supplementary Table S10).

Table 1 List of categories of differentially expressed genes in response to microgravity under different photoperiod

\begin{tabular}{|c|c|c|c|c|c|}
\hline \multirow[t]{2}{*}{ Group } & \multicolumn{2}{|c|}{$\begin{array}{l}\text { Transcriptional } \\
\text { behavior }\end{array}$} & \multicolumn{2}{|c|}{ Nu. of genes } & \multirow[t]{2}{*}{ Description } \\
\hline & LD $-\mu g$ & SD $-\mu g$ & WT & FG & \\
\hline$\mu g$-common & YES & YES & 1018 & 702 & $\begin{array}{l}\text { Genes differentially expressed (DE) and their } \\
\text { expression levels under SD- } \mu \text { g are similar to } \\
\text { those under LD- } \mu \mathrm{g}\end{array}$ \\
\hline \multirow[t]{3}{*}{$\mu g$-daylength-related } & YES & YES & 455 & 348 & $\begin{array}{l}\text { Genes DE under LD- } \mu \mathrm{g} \text { and SD- } \mu \mathrm{g} \text {, but with } \\
\text { different behavior }\end{array}$ \\
\hline & YES & NO & 2959 & 2872 & Genes DE only when $\mu \mathrm{g}$ is applied under LD \\
\hline & NO & YES & 1098 & 981 & Genes DE only when $\mu \mathrm{g}$ is applied under SD \\
\hline
\end{tabular}

181 To test whether there are potential common cis-acting elements among the

182 ' $\mu$ g-daylength-related' genes, we performed analyses using Plant Regulomics

183 (bioinfo.sibs.ac.cn/plant-regulomics) to find overrepresented motifs in the 1-kb

184 upstream sequence of the overrepresented genes in protein phosphorylation GO

185 terms with ' $\mu$ g-daylength-related' behaviors in WT and FG plants (Fig.6B and C;

186 Supplementary Table S10). Thirty-one of coregulated genes (Fig. 7B) were

187 identified to share four common motifs, of which occurrence were significantly high

188 as compared to that in random genomic regions (Fig. 7A). These include

$189 \mathrm{~A}(\mathrm{G} / \mathrm{T})$ ATTC, which is identical to the AtNIGT1/HRS1(AT1G13300) motif that is

190 present in the promoters of nitrate and phosphate signaling genes (Medice et al.,

191 2015), GAATATTC, which represents to KAN4 motif that provides boundary

192 maintenance and promotes the laminar growth of the inner ovule integument

193 (Gomez et al., 2016) and GGGACCAC, which is identifical to the transcription

194 factor TCP5 that controls plant thermomorphogenesis by positively regulating

195 PHYTOCHROME INTERACTING FACTOR 4(PIF4) activity (Han et al., 2019).

196 Furthermore, the AAAG, which is similar to the dof zinc finger protein MNB1A

197 element that has been suggested to regulate photosynthetic gene expression in Zea 
198 may (Cavalar et al., 2007). This result indicate that common regulators might be

199 involved in adaptation of plants to microgravity under different photoperiod

200 conditions.

Expression of FT could altered daylenth-related microgravity response of leaves To explain whether plants change response to microgravity after $F T$ expression under different daylength conditions, we compared expression of ' $\mu$ g-daylength related 'genes in FG with those in WT. The direction comparison of SD-1g genes in FG on ground with those in WT showed no significant correlation (Fig. 8A). In constrast, comparison of LD- $\mu$ g genes in FG with those in WT showed a strong positive correlation (Fig. 8B), while SD- $\mu$ g genes in FG showed a strong negative correlation with those in WT (Fig.8C), suggesting that the response of FG to microgravity was similar to those in WT under the LD, but different under under the SD, in which $F T$ had expressed by heating induction.

To further study response of plants to microgravity after $F T$ expression under the $\mathrm{SD}$, we focused on genes in three clusters. Cluster 1(C1) comprises 534 genes which expression in WT under both the LD and the the SD were modified as well as in FG under the LD, but unchanged in FG under the SD (Fig.8D and E ). Analysis of this cluster to assess overrepresented GO terms with the Biological Networks Gene Ontology tool (BINGO) indicated the link with cellular metabolic process, notably amino acid, amine, oxoacid metabolic process (Fig. 8F). Cluster 2(C2) is represented by 467 genes. Their expression did not change in WT under the LD and the SD as well in FG under the LD but was significantly up- or down-regulated in FG under the SD. BINGO analysis of the induced genes revealed that this cluster include many of the abotic stimulus response-associated genes noted in Fig.8G, as well as a number of genes involved in post-translational protein modification (Supplementary Table S9). Cluster 3 include 251 genes which expression altered in response to microgravity in both WT and FG under the LD, but didn't change in WT under the SD. BINGO analysis of this cluster genes suggested that the induced processes include metabolic process notably chlorophyll biosynthetic process, and 
227 response to stimulus, such as, high light, temperature and ethylene stimulus (Fig.

228 8H; Supplementary Table S9).

229 Impact of microgravity on daylength flowering pathways

230 To further explore the impact of microgravity on daylength flowering-time

231 pathways, we investigate expression pattern of the 49 core flowering control genes

232 (http://wikipathways.org) in response to microgravity in WT and FG under the LD

233 and the SD, respectively. Thirty-seven of them showed altered expression levels in

234 response to microgravity in WT and/or FG under at least one of daylength conditions (Supplementary Table S11). More than one-third of these core flowering control genes are circadian clock genes, including, LATE ELONGATED HYPOCOTYL (LHY), REVEILLE 1(RVE1), RVE2, CLOCKASSOCIATED1 (CCA1), PATHOGEN AND CIRCADIAN CONTROLLED 1(PCC1), EARLY FLOWERING 4(ELF4), ELF4-L4, GIGANTEA(GI), CONSTITUTIVE PHOTOMORPHOGENIC1(COP1), CONSTANS (CO), CONSTANS-LIKE9(COL9), ARABIDOPSIS PSEUDO-RESPONSE REGULATOR 5(APRR5). The differential expression of these core flowering control genes between WT and FG in response to microgravity was observed (Fig.9A and B). For example, expression of $P C C 1$ is down-regulated in FG on ground, while up-regulated in space, in comparison with that in WT. Expression of FT in FG were apparent higher under both LD and SD conditions on ground and in space than that in WT(Fig. 9A), consistenting with GFP signal in FG observed by inflight images (Fig. 3P). The gene $C O$, which is necessary for the daylength regulating of flowering, exhibited up-regulated by 4.5-fold in WT under LD, while down-regulated by 0.1-fold in WT under SD in space in comparison with their ground controls. By contrast, FG showed no change of CO expression to microgravity under both LD and SD conditions(Supplementary Table S11). In addition, several circadian clock genes were abserved among FT interactome (Fig. 9 B). REV2, which is involved in regulating both photoperiod pathway and circadian processes, was up-regulated about 115 fold in FG and about 35 fold in WT under the LD in space in comparison with their control on ground, 
256 but less increased under the SD (Fig.9C). This indicates a specific role of REV2 in

257 regulating microgravity response under the LD condition in space. Expression level

258 of $L H Y$ exhibited up-regulated under LD in WT and FG in space (5.82- and

259 15.56-fold, respectively), but more significantly increased under SD in space (9.05-

260 and 26-fold, respectively). In contrast, expression level of GI was down-regulated

261 under both LD and SD in WT and FG in space (Fig.9 C). These results indicated

262 that circadian clock gene could play an important role for plant adaptation to

263 microgravity in space during flowering.

\section{Discussion}

265 Day length and microgravity are both impact on growth and development of plants 266 in space, but interaction between them remain unclear. In this study, we constructed 267 a transgenic plants (pHSP::FT; pHSP::GFP) and setup a protocol to initiate the 268 floral transition on orbital condition using HS treatment by remote control. Using 269 this experimental system, we contributed to identify: (1) plant response to 270 microgravity at transcriptional levels depend on the daylength conditions. (2) 271 daylength-related microgravity response could be involved in alteration of 272 transcriptional activity in protein sythesis and post-translation protein modulation, 273 notably protein phosphorlation. (3) Expression of $F T$ in Arabidopsis leaves by HS 274 induction can change the response of plant to microgravity under the SD, possibly 275 through modulating expression of circadian clock genes in photoperiod controlling 276 flowering pathway.

277 Manipulate de novo transgene expression at key developmental stage using a gene 278 switch, which can mediate an "on/off" situation of gene activity, will be very 279 importance to design efficient production and resilient crop cultivation in 280 bioregenerative life-support system (BLSS) for a long-term mission such as on earth 281 orbit space station, moon and Mars in the future. A pervious study using transgenic 
282 Arabidopsis containing the alcohol dehydrogenase (Adh) gene promoter linked to 283 the $\beta$-glucuronidase (GUS) reporter gene has been used to evaluate the stress signal 284 perception and transduction in Arabidopsis in spaceflight (Paul et al., 2001). In this 285 study, we demonstrate that a HSP17.4 promoter combined with FT and GFP could 286 be used in flowering induction of transgenic Arabidopsis by simply heating the 287 manipulate crops for high production in space through controlling plant flowering 290 time.

291 Flowering is a prerequisite for crop production whenever seeds or fruits are harvested (Blümel et al., 2015; Shim et al., 2017). Various environmental stresses, such as drought, salt and temperature, were reported to interfered flowering

294 (Galbiati et al., 2016; Fernández et al., 2016). Microgravity is a novel stress to 295 plants, which were evolved on earth (Hampp et al., 1997; Zheng et al., 2008; Zhang 296 and Zheng, 2015; Paul et al., 2017; Wu et al., 2020). Previous studies indicated that microgravity on spaceflight is a compound stress, imposing multiple constraints on plants by interation with other environmental factors. For example, roots of plants in space appeared to become hydortropically more sensitive to moisture gradients

300 (Morohashi et al., 2017) and altered response to red- and blue-light phototropism 301 (Valbuena et al., 2018; Herranz et al., 2019). In this study, we found that 302 Arabidopsis plants grown under the LD exhibited more sensitive to microgravity in comparison those under the SD condition at transcriptional level. Down-regulation 304 of expression in ribosome biogenesis and RNA processing (i.e. ncRNA, rRNA 305 metabolism and processing) and amino acid metabolic process was observed among 306 ' $\mu \mathrm{g}$-common' genes as well as ' $\mu \mathrm{g}$ - daylength related ' genes. This result is 
consistent with that regulation of ribosome biogenesis, which is linked to factors controlling cell growth and proliferation, was decreased in Arabidopsis cell cultures and seedlings in real or simulated microgravity(Matía et al., 2010; Manzano et al., 2012; Kamal et al., 2018). The reason for down-regulation of ribosome biogenesis under microgravity is unknown. A pervious study estimated that cells dedicate $\sim 80 \%$ of total trancriptional activity to the synthesis of rRNAs and proteins for ribosome biogenesis (Wamer, 1999), making ribosome biogenesis a major nutrient and energy-consuming process in growing cells (Lempiäinen and shore, 2009). Down-regulation of ribosome biogenesis found in ' $\mu$ g-common' genes suggest a limitation in nutrient and energy supply to plants during the microgravity response under both the LD and the SD condition. Another interesting finding is our observation that microgravity trigger networks related to daylength signals altered expression of genes involved in the GO category 'protein phosphorylation' in both the WT and FG. These ' $\mu$ g-daylength-related' genes, including protein kinase, receptor-like protein kinase, phosphatase and signaling in light, ethylene and calcium, were down-regulated expression in the $\mu \mathrm{g}-\mathrm{LD}$ datasets and/or up-regulated expression in the $\mu \mathrm{g}-\mathrm{SD}$ datasets. This result is consistent with a recently study, which indicated that protein phosphorylation plays a crucial role in gravisignaling, and gravitropism and phototropism of plants (Yang et al., 2020). Among 'protein phosphorylation' GO category genes, we found phytochrome interacting factor 4(PIF4) and phototropic-responsive $N P H 3$ family protein, and a blue light receptor phototropin 1(PHOT1), which showed expression profiles in response to microgravity were modulated different by day length. Similarly, expression of genes involved in sensing the extracellular environment and triggering intracellular signals, such as several wall-associated kinase (WAK), ethylene response sensors and 
calcium-dependent protein kinases, were mostly downregulated in response to microgravity under the LD only, but didn't change expression level under the SD (Supplemantary Table S10).

Plant photoperiodic regulation can be divided into three parts: light input, circadian clock, and output. Previous study pointed that light-associated pathways in Arabidopsis showed significant down-regulation in microgravity (Valbuena et al., 2018; Vandenbrink et al., 2019). However, those experiments were all performed under the LD condition, but no one under the SD. Our study found that light-associated genes, such as PHOT1 was down-regulated only under the LD, while expression of PIF4 and NPH3 didn't changed under the LD, but up-regulated specifically under the SD, suggesting that photoperiod apparently affect plant response to microgravity during light input. In addition, light information is integrated into innate photoperiodic timing mechanisms governed by the circadian clock to induce $F T$ expression that trigger flowering (Shim et al., 2017). The plant circadian clock consists of multiple transcription-translation feedback loops that are influenced by environmental signals, linking the clock with plant stress adaptation. For example, phytochrome- and cryptochrome-mediated light signals mediate the induction of CCA1, LHY, and PRR9 gene expression(Somers et al., 1998; Farré et al., 2005; Bieniawska et al., 2008). The expression of circadian clock components, such as, CCA1, ELF3, GI, GRP7, PRR9, TOC1, and ZTL, were affected by stress environment, including water use efficience of Arabidopsis (Simon et al., 2020), high light (Yakir et al., 2007) as well as the 3-D clinostat rotational simulated microgravity (Xie and Zheng, 2020). In this study, our data indicate that the circadian oscillator is important for regulating microgravity response of Arabidopsis. Up-regulation of morning components (CCA1, LHY, REV1 and REV2) and 
down-regulation of late day $(G I)$ and evening (ELF4-L4) components of the

358 circadian oscillator in space under the LD and/or the SD in both WT and FG.

359 Additionally, COL9 and COP1 enhanced expression levels in response to

360 microgravity specifically under the LD condition, while APRR5 increase expression

361 levels in response to microgravity specifically under the SD condition. Together,

362 this results suggests that the circadian oscillator can be altered expression level in

363 response to microgravity dependent on daylength condition, which could in

364 particular interfere with flowering in space. Change circadian function by

365 microgravity in space influence plant flowering and fitness offerig the hypothesis

366 that optimizing circadian function will enhance crop productivity in space. In the

367 future, more refinement of our understanding of the circadian clock mechanism

368 under different photoperiodic conditions in space is necessary to inform

369 manipulation towards the goal of enhacing crop productivity in bioregenerative

370 life-support systems (BLSS).

$371 \quad$ Methods

\section{$372 \quad$ Plant Materials and growth condition}

373 Arabidopsis thaliana Columbia (Col-0) ecotype was used as the wild-type. Plants

374 were germinated and grown in plastic cups under long-day (16h light $/ 8 \mathrm{~h}$ dark) at $375120 \mu \mathrm{mol} . \mathrm{m}^{-2} \cdot \mathrm{s}^{-1}$ conditions for 5 days, then set in the root modules $(240 \times 120 \times 65$

$376 \mathrm{~mm}^{3}$ ) containing a commercially available vermiculite immersed by a medium

377 containing MS macronutrients (Murashige and Skoog, 1962 ) and cultived in 378 greenhouse for 20 days prior to flight.

\section{Construction of transgenic Arabidopsis plants}

380 For the construction of $p H S P:: F T$, the coding sequence (CDS) of $F T$ was amplified 
381 by PCR from a Col-0 cDNA using the primers with the restriction sites underlined 5'-ATCACTAGTATGTCTATAAATATAAGAGACCCTCTTA-3' and 5'CGTTCTAGACTAAAGTCTTCTTCCTCCGCAGC-3' and ligated into a pBluescript KS minus vector. A 1109 bp DNA fragment, upstream from HSP17.4(AT3G46230) start codon corresponsing to the putative promoter, was amplfied

by

PCR

with

the

primers

5'-ATGACTAGTCGTTTCGCTTACTCTGTTTGC-3', and fused to the FT coding

sequence in the pBluescript KS minus vector. For the construction of $p H S P:: G F P$, the HSP17.4 promoter DNA fragment was fuse to a GFP in pBluescript SK minus vector. $p H S P:: F T$ or $p H S P:: G F P$ were then cleaved and ligated into the pCambia1301-NOS-3, vector. These two gene fusions were transferred to Arabidopsis (Col-0) plants through the Agrobacterium tumefaciens strain GV3101, respectively, by the floral dip method according to the methods of Clough and Bent (1998). After regeneration in the presence of hygromycin, transgenic plants were $p H S P:: F T, p H S P: G F P(\mathrm{FG})$ gene were co-expression in Arabidopsis by genetic crossing as described by Qi and Zheng (2013). F3 progeny homozyous for FG were used for space expriments.

\section{Hardware design and the spaceflight experiment}

The plant growth system used for the SJ-10 experiment consisted of four growth compartments, illumination, photograph, air-flowing heating and humidity controling system (Fig. 1A-C). Plants have about 4-6 rosette leaves when they loaded into the growth chamber (Fig. 1D). Two set of plants were prepared and placed in the plant growth units (PGUs) for spaceflight experiment (Fig.1A, B and 
406 C) and the ground control, respectively. The flight PGU was positioned in the 407 capsual of satellite about $8 \mathrm{~h}$ prior to launch. The SJ-10 satellite was in orbit for 408 about 12 days and 15h (lauch: 1:38, April 6, 2016; landing: 16:30, April 18, 2016). 409 Illumination was provided by light banks made up of 200 solid state light emitting 410 diode (LED) lamps (400-700 nm white light and red light, 2:1) on a long-day (LD, 411 16h light/8h dark) or a short-day (SD, 8h light/16h dark) photoperiod. Inside the 412 chambers, temperatures were $22 \pm 2^{\circ} \mathrm{C}$, relateve humidity was between $90 \%$ and $413100 \%$. The photosynthetically active photon flux density produced by LED lamps 414 was $120 \mu \mathrm{mol} \cdot \mathrm{m}^{-2} \cdot \mathrm{s}^{-1}$ for Arabidopsis at surface of the first leaf of the experimental 415 plants. Temperature and humidity were recorded every 1 min during flight. These 416 data were used to set the ground control in a control growth chamber. Three video 417 cameras were mounted in the PGU to allow recording of plant growth and 418 development plants in space. Photographic equipments were consisted of two video cameras (image size $1280 \times 1024$ pixels) and one GFP fluorescence camera, which were automatic and preprogrammed and allowed recording of plants in PGUs both 421 in visible light and in GFP fluorescence (Fig.1B and C). The photographs were taken at 2-h intervals during the light period. Two video cameras were used for 423 photographed seedlings grown under the LD and the SD conditions, respectively. 424 The GFP fluorescence camera was used to follow expression of GFP in seedlings after heating induced. All manipulations involved in the experiment were automated or carried out by remote control. After SJ-10 satellite return to Earth, the PGU was unloaded and received at a 428 temporary laboratory in the landing site about $2 \mathrm{~h}$ post-landing. Plants were harvested and fixed with RNAlater solution (ambion, Austin, TX, USA) at the 430 landing site. The samples were then brought to our Shanghai laboratory where they 
were analyzed for transcriptional changes.

\section{Sample processing and Analysis}

433 Total RNA was extracted from the space samples and the ground controls and then 434 purified using miRNeasy Mini Kit (Cat\#217004, QIAGEN, GmBH, Germany) 435 following the manufacturer's instructions and checked for a RIN number to inspect 436 RNA integration by an Agilent Bioanalyzer 2100 (Agilent technologies, Santa Clara, 437 CA, US). RNA amplified, labeled and purified by using GeneChip 3' IVT PLUS 438 Reagent Kit (Cat\#902416, Affymetrix, Santa Clara, CA, US) following the 439 manufacturer's instructions to obtain biotin labeled cRNA. Array hybridization and 440 wash was performed using GeneChip ${ }^{\circledR}$ Hybridization, Wash and Stain Kit 441 (Cat\#900720, Affymetrix, Santa Clara, CA, US) in Hybridization Oven 645 442 (Cat\#00-0331-220V, Affymetrix, Santa Clara, CA, US) and Fluidics Station 450 443 (Cat\#00-0079, Affymetrix, Santa Clara, CA, US) following the manufacturer's 444 instructions.

\section{Analysis of microarray data}

446 Slides were scanned by GeneChip ${ }^{\circledR}$ Scanner 3000 (Cat\#00-00212, Affymetrix, 447 Santa Clara, CA, US) and Command Console Software 4.0 (Affymetrix, Santa Clara, 448 CA, US) with default settings. Raw data were normalized by MAS 5.0 algorithm, 449 Affymetrix packages in R. Probe sets with signal values lower than the detectable 450 range were adjusted to 75 and probe sets with the values of 75 for all conditions were removed from subsequent analysis. The averages of normalized ratios are

452 calculated by dividing the average of normalized signal channel intensity by the 453 average of the normalized control channel intensity. The standard deviation of the 454 ground control (two biological replicates) was employed to identify genes of 
significant changes relative to the ground controls ( $\mathrm{P}$ value $<0.05)$. Only genes that

456 showed transcript level changes in at least two folds in comparison with its ground

457 control and with the same tendency in both biological replicates were considered as

458 relevant for microgravity. Gene Ontology (GO) Overrepresentation was performed

459 using PANTHER (Fisher's Exact type with False Discovery Rate correction)

460 (http://www.pantherdb.org) (Mi et al. 2019). For motif enrichment, motifscan (Sun

461 et al., 2018 ) was used to determine whether the occurrence of a given motif in input

462 genes was significantly high as compared to that in random regions (Ran et al., 463 2019).

\section{$464 \quad$ Real-time RT-PCR}

465 Total RNA was extracted from leaves of the space samples and the ground controls 466 as described. The genes and their qRT-PCR primers are presented in Supplementary 467 Table S1. The Arabidopsis ACTIN gene was used as the loading control for all 468 qRT-PCRs. At least three technical replicates of each biological replicate were used 469 for real-time PCR analysis.

\section{Acknowledgments}

The authors are indebted to Professor W.R. Hu for valuable and helpful suggestions and Professor T. Zhang's group for PGU construction and helping in the space experiment. This work was supported by the China Manned Space Flight Technology project Chinese Space Station, the National Natural Science Foundation of China (31870850), the Strategic Pioneer Projects of CAS (XDB37020104), the National natural fund joint fund project (U1738106).

Author Contributions: LW and JX carried out plant culture, data curation and investigated gene expression; YW, CM, YJ and YD participated in investigation, $\mathrm{HZ}$ 
conceived of the study, participated in its design and coordination, and drafted the manuscript. All authors have read and agreed to the published version of the manuscript.

Conflicts of Interest: The authors declare that they have no conflict of interest.

\section{Referneces}

Achard, P., Cheng, H., De Grauwe, L., Decat, J., Schoutteten, H., Moritz, T., Van Der Straeten, D., Peng, J.\& Harberd, N.P. Integration of plant responses to environmentally activated phytohormonal signals. Science. 311, 91-94 (2006).

Agliassa, C., Narayana, R., Bertea, C.M., Rodgers, C.T.\&Maffei, M.E. Reduction of the geomagnetic field delays Arabidopsis thaliana flowering time through downregulation of flowering-related genes. Bioelectromagnetics 39, 361-374(2018).

Becker, B, Holtgrefe, S., Jung, S., Wunrau, C., Kandlbinder, A., Baier, M., Dietz, K-J., Backhausen, J.E.\& Scheibe, R. Influence of the photoperiod on redox regulation and stress responses in Arabidopsis thaliana L.(Heynh.) plants under long- and short-day conditions. Planta 224, 380-393(2005).

Bieniawska, Z., Espinoza, C., Schlereth, A., Sulpice, R., Hincha, D.K.\&Hannah, M.A. Disruption of the Arabidopsis circadian clock is responsible for extensive variation in the cold-responsive transcriptome. Plant Physiol. 147, 263-279(2008).

Blümel, M., Dally, N. \&Jung, C. Flowering time regulation in crops-what did we learn from Arabidopsis? Curr.Opin.Biotechnol. 32,121-129 (2015).

Boyes, D.C., Zayed, A.M., Ascenzi, R., McCaskil,l A.J., Hoffman, N.E., Davis, K.R., Görlach, J. Growth stage-based phenotypic analysis of Arabidopsis: a model for high throughput functional genomics in plants. Plant Cell 13, 1499-1510 (2001).

Campbell, W.F., Salisbury, F.B., Bugbee, B., Klassen, S., Naegle, E., Strickland, D.T., Bingham, G.E., Levinskikh, M., Iljina, G.M., Veselova, T.D., Sytchev, V.N., Podolsky, G., McManus, W.R., Bubenheim, D.L., Stieber, J., \& Jahns, G. Comparative floral development of Mir-grown and ethylene-treated, Earth-grown Super Dwarf wheat. J. Plant Physiol.158,1051-1060 (2001).

Cavalar, M., Phlippen, Y., Kreuzaler, F.\& Peterhänsel, C. A drastic reduction in DOF1 transcript levels does not affect $\mathrm{C} 4$-specific gene expression in maize. J Plant Physiol. 164,1665-1674 (2006).

Czarnecka, E., Fox, P.C.\&Gurley, W.B. In Vitro interaction of nuclear proteins with the promoter of soybean heat shock gene Gmhsp17.5E. Plant Physiol. 94:935-43(1990). 
De Micco, V., De Pascale, S., Paradiso, R.\&Aronne, G. Microgravity effects on different stages of higher plant life cycle and completion of the seed-to-seed cycle. Plant Biol. 16: 31-38 (2014).

Farré, E.M., Harmer, S.L., Harmon, F.G., Yanovsky, M.J.\& Kay, S.A. Overlapping and distinct roles of PRR7 and PRR9 in the Arabidopsis circadian clock. Curr Biol. 15:47-54.(2005).

Fernández, V., Takahashi, Y., Gourrierec, J.L., Coupland, G. Photoperiodic and thermosensory pathways interact through CONSTANS to promote flowering at high temperature under short days. Plant J. 86, 426-440 (2016).

Galbiati, F., Chiozzotto, R., Locatelli, F., Spada, A., Genga, A.\& Fornara, F. Hd3a, RFT1 and Ehd1 integrate photoperiodic and drought stress signals to delay the floral transition in rice. Plant Cell Environ. 39,1982-1993(2016).

Gomez, M. D., Ventimilla, D., Sacristan, R., \& Perez-Amador, M. A. Gibberellins regulate ovule integument development by interfering with the transcription factor ATS. Plant physiol. 172, 2403-2415(2016).

Hampp, R., Hoffmann, E., Schonherr, K., Johann, P., Filippis, L.E. Fusion and metabolism of plant cells as affected by microgravity. Planta 203,S42-S53 (1997).

Han, X., Yu, H., Yuan, R., Yang, Y., An, F.\& Qin, G. Arabidopsis Transcription Factor TCP5 Controls Plant Thermomorphogenesis by Positively Regulating PIF4 Activity. iScience 15, 611-622(2019).

Herranz, R., Vandenbrink, J. P., Villacampa, A., Manzano, A., Poehlman, W. L.\&Feltus, F. A., et al. RNAseq analysis of the response of Arabidopsis thaliana to fractional gravity under blue-light stimulation during spaceflight. Front. Plant Sci. 10, 1529 (2019).

Hoson, T., Soga, K., Wakabayashi, K., Hashimoto, T., Karahara, I., Yano, S., Tanigaki, F., Shimazu, T., Kasahara, H., Masuda, D., Kamisaka, S. Growth stimulation in inflorescences of an Arabidopsis tubulin mutant under microgravity condition in space. Plant Biol. 16, 91-96 (2014).

Kamal, K.Y., Herranz, R., van Loon, J.J.\&Medina, F.J. Simulated microgravity, Mars gravity, and $2 \mathrm{~g}$ hypergravity affect cell cycle regulation, ribosome biogenesis, and epigenetics in Arabidopsis cell cultures. Sci Rep. 8, 6424 (2018).

Karahara, I., Suto, T., Yamaguchi, T., Yashiro, U., Tamaoki, D., Okamoto, E., Yano, S., Tanigaki, F., Shimazu, T., Kasahara, H., Kasahara, H., Yamada, M., Hoson, T., Soga, K.\& Kamisaka, S. Vegetative reproductive growth of Arabidopsis under microgravity conditions in space. J. Plant Res. 133, 571-585(2020).

Kiss, J.Z., Brinckmann, E., Brillouet, C. Development and growth of several strains 
of Arabidopsis seedlings in microgravity. Int. J. Plant Sci. 161, 55-62 (2000).

Krikorian, A.D. \&O'Connor, S.A. Karyological observations. Ann. Bot. 54, 49-63(1984)..

Kuang, A., Musgrave, M.E.\& Matthews, S.W. Modification of reproductive development in Arabidopsis thaliana under spaceflight conditions.Planta 198:588-94(1996).

Kwon, T., Alan Sparks, J., Nakashima, J., Allen, S. N., Tang, Y., \& Blancaflor, E.B. Transcriptional response of Arabidopsis seedlings during spaceflight reveals peroxidase and cell wall remodeling genes associated with root hair development. Am. J. Bot. 102, 21-35(2015).

Lee, J.H., Lee, J.S. \& Ahn, J.H. (2008). Ambient temperature signaling in plants: an emerging field in the regulation of flowering time. J. Plant Biol. 51, $321-326$.

Lempiäinen, H.\&Shore, D. Growth control and ribosome biogenesis. Curr. Opin. Cell Biol. 21, 855-63 (2009).

Levinskikh, M.A., Sychev, V.N., Derendyaeva, T.A., Signalova, O.B., Salisbury, F.B., Campbell, W.F., Bingham, G.E., Bubenheim, D.L.\&Jahns, G. Analysis of the spaceflight effects on growth and development of Super Dwarf wheat grown on the Space Station Mir. J. Plant Physiol. 156, 522-9(2000).

Link, B.M., Durst, S.J., Zhou, W.\& Stanković, B. Seed-to-seed growth of Arabidopsis thaliana on the international space station. Adv. Space Res. 31, 2237-2243(2003).

Link, B.M., Busse, J.S.\&Stanković, B. Seed-to-seed-to-seed growth and development of Arabidopsis in microgravity. Astrobiology 14, 866-875(2014).

Ma, Y., Shabala, S., Li, C., Liu, C., Zhang, W.\& Zhou, M. Quantitative trait loci for salinity tolerance identified under drained and waterlogged conditions and their association with flowering time in barley (Hordeum vulgare. L). PLoS One. 10, e0134822 (2015).

Manzano, A.I., van Loon, J.J., Christianen, P.C., Gonzalez-Rubio, J.M., Medina, F.J.\&Herranz R. Gravitational and magnetic field variations synergize to cause subtle variations in the global transcriptional state of Arabidopsis in vitro callus cultures. BMC Genomics 13,105 (2012).

Matía, I., González-Camacho, F., Herran,z R., Kiss, J.Z., Gasset, G., van Loon, J.J., Marco, R.\& Medina, F.J. Plant cell proliferation and growth are altered 
by microgravity conditions in spaceflight. J. Plant Physiol. 167, 184-93 (2010).

Medici, A., Marshall-Colon, A., Ronzier, E., Szponarski, W., Wang, R., Gojon, A., Crawford, N.M., Ruffel, S., Coruzzi, G.M.\&Krouk G. AtNIGT1/HRS1 integrates nitrate and phosphate signals at the Arabidopsis root tip. Nat. Commun. 6, 6274 (2015).

Mi, H., Muruganujan, A., Huang, X. et al. Protocol Update for large-scale genome and gene function analysis with the PANTHER classification system (v.14.0). Nat Protoc 14, 703-721(2019).

Morohashi, K., Okamoto, M., Yamazaki, C., Fujii N., Miyazawa Y., et al. Gravitropism interferes with hydrotropism via counteracting auxin dynamics in cucumber roots: clinorotation and spaceflight experiments. New Physiolist 215,1476-1489 (2017).

Murashige, T.\& Skoog, F. A revised medium for rapid growth and bio assays with tobacco tissue culture. Physiol. Plant. 15, 473-497(1962).

Nechitailo, G.S.\& Mashisky, A.L. Space biology: studies at orbital station. Moscow, Mir Publishers. (1993)

Paul, A.L., Zupanska, A.K., Ostrow, D.T., Zhang, Y., Sun, Y., Li, J.L., Shanker, S., Farmerie, W.G., Amalfitano, C.E.\& Ferl, R.J. Spaceflight transcriptomes: unique responses to a novel environment. Astrobiology 12, 40-56(2012).

Paul, A.L., Daugherty, C.J., Bihn, E.A., Chapman, D.K., Norwood, K.L.\& Ferl, R.J. Transgene expression patterns indicate that spaceflight affects stress signal perception and transduction in Arabidopsis. Plant Physiol. 126, 613-621(2001).

Paul, A. L., Sng, N. J., Zupanska, A. K., Krishnamurthy, A., Schultz, E. R.\& Ferl, R. J. Genetic dissection of the Arabidopsis spaceflight transcriptome: are some responses dispensable for the physiological adaptation of plants to spaceflight? PloS One 12, e0180186 (2017).

Posé, D., Verhage, L., Ott, F., Yant, L., Mathieu, J., Angenent, G.C., Immink, R.G.H.\& Schmid, M. Temperature-dependent regulation of flowering by antagonistic FLM variants. Nature 503, 414-417(2013).

Qi B.\&Zheng H. Modulation of root-skewing responses by KNAT1 in Arabidopsis thaliana. Plant J. 76:380-392(2013).

Ran, X., Zhao, F., Wang, Y., Liu, J., Zhuang, Y., Ye, L., Qi, M., Cheng, J.\& Zhang, Y. Plant regulomics: A data-driven interface for retrieving upstream 
regulators from plant multi-omics data. Plant J. doi: 10.1111/tpj.14526 (2019).

Rasmussen, O., Baggerud, C., Larssen, H.C., Evjen, K.\& Iversen, T-H. The effect of 8 days of microgravity on regeneration of intact plants from protoplasts. Physiol. Plant. 92, 404-411(1994).

Sherrard, M.E.\& Maherali, H. The adaptive significance of drought escape in Avena barbata, an annual grass. Evolution 60, 2478-2489(2006).

Shim, J.S., Kubota, A.\& Imaizumi T. Circadian clock and photoperiodic flowering in Arabidopsis: CONSTANS is a hub for signal integration. Plant Physiol. 173:5-15 (2017).

Simon, N.M.L., Graham, C.A., Comben, N.E., Hetherington, A.M., Dodd, A.N. The circadian clock influences the long-term water use efficiency of Arabidopsis. Plant Physiol. 183, 317-330(2020).

Soga, K., Wakabayashi, K., Hoson, T.\& Kamisaka, S. Inhibition of reproductive growth of Arabidopsis in airtight vessels. Adv. Space Res. 23, 2037-40(1999).

Soga, K., Wakabayashi, K., Kamisaka, S.\& Hoson, T. Stimulation of elongation growth and xyloglucan breakdown in Arabidopsis hypocotyls under microgravity conditions in space. Planta 215,1040-1046(2002).

Somers D.E., Devlin P.F.\& Kay S.A. Phytochromes and cryptochromes in the entrainment of the Arabidopsis circadian clock. Science. 282,1488-1490(1998).

Strickland, D.T., Campbell, W.F., Salisbury\& F., Bingham, G.E. Morphological assessment of reproductive structures of wheat grown on Mir. Grav. Space Biol. Bull. 11,14(1997).

Stankovic, B. A. plant space odyssey. Trends Plant Sci. 6, 591-593(2001).

Sugimoto, M., Oono, Y., Gusev, O., Matsumoto, T., Yazawa, T., Levinskikh, M.A., Sychev, V.N., Bingham, G.E., Wheeler, R.\& Hummerick M. Genome-wide expression analysis of reactive oxygen species gene network in Mizuna plants grown in long-term spaceflight. BMC Plant Biol. 14, 4(2014).

Sun, H., Wang, J., Gong, Z., Yao J., Wang Y., Xu J., Yuan G., Zhang Y.\& Shao Z. Quantitative integration of epigenomic variation and transcription factor binding using MAmotif toolkit identifies an important role of IRF2 as transcription activator at gene promoters. Cell Discov. 4, 38(2018). 
Sychev, V.N., Levnskikh, M.A., Gostimsky, S.A., Bingham, G.E.\& Podolsky, I.G. Spaceflight effects on consecutive generations of peas grown onboard the Russian segment of the International Space Station. Acta Astron. 60, 426-432(2003).

Tripathy, B. C., Brown C. S., Levine H. G.\&Krikorian A. D. Growth and photosynthetic responses of wheat plants grown in space. Plant Physiol. 110, 801-806(1996).

Ueda, J., Miyamoto, K., Yuda, T., Hoshino, T., Sato, K., Fujii, S., Mukai, C., Kamigaichi, S., Izumi, R., Ishioka, N., Aizawa, S., Yoshizaki, I., Shimazu, T.\&Fukui K. STS-95 space experiment for growth and development, and auxin polar transport. Biol. Sci. Space 14, 47-57(2000).

Valbuena, M.A., Manzano, A., Vandenbrink, J.P., Pereda-Loth, V., Carnero-Diaz, E., Edelmann, R.E., Kiss, J.Z., Herranz, R.\& Medina F.J. The combined effects of real or simulated microgravity and red-light photoactivation on plant root meristematic cells. Planta 248, 691-704(2018).

Vandenbrink, J. P.,Herranz, R., Poehlman,W. L., Feltus, F.A., Ciska, M.,Medina, F. J.,et al. RNA-seq analyses of Arabidopsis thaliana seedlings after exposure to blue-light phototropic stimuli in microgravity. Am. J. Bot. 106, 1466-1476(2019).

Wang, L., Han, F.\& Zheng, H. Photoperiod-controlling guttation and growth of rice seedlings under microgravity on board Chinese spacelab TG-2. Microgravity Sci. Tech. 30, 834-847(2018).

Warner J. R. The economics of ribosome biosynthesis in yeast.Trends Bioch. Sci. 24, 437-440(1999).

Wu Y., Xie J., Wang L.\& Zheng H. Circumnutations and growth of inflorescence stems of Arabidopsis thaliana in response to microgravity under different photoperiod conditions. Life 10, 26 (2020).

Xie, J.\& Zheng H. Arabidopsis flowering induced by photoperiod under 3-D clinostat rotational simulated microgravity. Acta Astro. 166,567-572 (2020) .

Yakir E., Hilman D., Hassidim M.\&Green R.M. CIRCADIAN CLOCK ASSOCIATED1 transcript stability and the entrainment of the circadian clock in Arabidopsis. Plant Physiol. 145, 925-932 (2007).

Yang, Y., Al-Baidhani, H.H.J., Harris, J., Riboni, M., Li, Y., Mazonka, I., Bazanova, N., Chirkova, L., Hussain, S., Hrmova, M., Haefele, S., Lopato, S., \& 
Kovalchuk, N. DREB/CBF expression in wheat and barley using the stress-inducible promoters of HD-Zip I genes: impact on plant development, stress tolerance and yield. Plant Biotechnol. J. 18, 829-844(2020).

Zhang, Y., Wang, L., Xie, J.\& Zheng H. Differential protein expression profiling of Arabidopsis thaliana callus under microgravity on board the Chinese SZ-8 spacecraft. Planta 241, 475-488(2015).

Zhang Y.\&Zheng H. Changes of protein expression in plastid and mitochondria of Arabidopsis thaliana callus on board Chinese spacecraft SZ-8. Microgravity Sci. Tech. 27, 387-401(2015).

Zheng H. Flowering in space. Microgravity Sci. Tech. 30, 783-791(2018).

Zheng H., Wang H., Wei N., Chen A., Wang L., Zheng W.\& Zhang T. Live imaging technique for studies of growth and development of Chinese cabbage under microgravity in a recoverable satellite (SJ-8). Microgravity Sci. Tech. 20, 137-143(2008). 


\section{Figure legends}

Figure 1 Experimental setup on board the Chinese recoverable satellite SJ-10.

(A) The external view of the plant growth unit (PGU) $(\mathrm{H} \times \mathrm{W} \times \mathrm{D}=370 \times 270 \times 270 \mathrm{~mm})$ with the cover.

(B) The inside view of the PGU without the cover, as described in (C).

(C) Diagram of PGU used on board the Chinese satellite SJ-10. Showing the distribution and state of components and samples in PGU during space flight. The components included four root modules, three cameras, two fans and heating parts, and four LED banks with controlled long-day (LD) or short-day (SD) photoperiods, respectively. indicating LED plates; heating systems; video CCD cameras(Cam 1 and Cam 2); the GFP imaging camera (Cam f) and root modules (R1 and R3 with rice seedlings; R2 and R4 with Arabidopsis seedlings).

(D)An overview of the time process of the space experiment. wild-type and transgenic Arabidopsis plants were germinated and grown in the root module in green house on ground for $20 \mathrm{~d}$ after sowing. At this age, the plants had formed four rosette leaves, when they were loaded into PGU less than $24 \mathrm{~h}$ prior to take off. Heating treatment (red arrows point) to activated expression of $\mathrm{pHSP}:: F T$; $\mathrm{pHSP}:: G F P$ was performed at day 2 on orbiter. The plants in PGU were grown under long-day (LD, $16 \mathrm{~h}$ light/8 h dark) and short-day(SD, $8 \mathrm{~h}$ light/16 h dark) conditions in space $(\mu \mathrm{g})$ and on ground $(\mathrm{g})$, respectively.

(E and F) 20-day old wild-type (WT) and transgenic ( $p H S P:: F T ; p H S P:: G F P, \mathrm{FG}$ ) Arabidopsis seedlings grown in the root modules before being loaded into the PGU.

( $\mathrm{G}$ and $\mathrm{H}$ ) The situation of samples recovered from spaceflight under LD $(\mathrm{G})$ and SD $(\mathrm{H})$ conditions. Bars $=1 \mathrm{~cm}$.

Figure 2 Floral transition time of wild-type and $p H S P:: F T$; $p H S P:: G F P$ transgenic plants grown under the long-day condition on ground and in flight on board the SJ-10 satellite. 
(A) example images of plants grown under long-day condition on ground(1g, on ground) and in space ( $\mu \mathrm{g}$, in space) at day 2 (d2) to day 9 (d9) after satellite took off.

(B and C) comparison of floral transition time and average length of stems of wild-type (WT) and $p H S P:: F T ; p H S P:: G F P(\mathrm{FG})$ transgenic plants under the long-day (LD) in space with their controls on ground. Data are determined from the living images of plants on orbit in space or on ground. $n=4$ plants per treatment.

(D) example images of plants of wild-type and transgenic plants under the short-day (SD) condition on ground and in space at $\mathrm{d} 2$ to $\mathrm{d} 9$ after satellite took off.

(E and F) comparison of floral transition time and average length of stems of WT and FG plants treated by heating shock (red asteres indicate) under the SD condition in space and on ground. Data are determined from the living images downloaded from the plant growth units in space and on ground. $n=4$ plants per treatment. Bars in $(A)$ and $(D)=10 \mathrm{~mm}$.

Figure 3 GFP expressing in short-day grown plants treated by $37^{\circ} \mathrm{C}$ heating on board SJ-10.

(A) $p H S P:: F T$; $p H S P:: G F P$ transgenic plants and wild-type (WT) plants photographed in white light before launching. The framed region in (A) is detailed in (B).

(C) a represent GFP image showed the expression of GFP of leaves1-6 in (B)

(D-O) Example GFP images were captured by the GFP imager at day 2 to day 4 after heating treatment. The images were captured at four time points (15:50, 15:55, 23:50 and 23:55, on board SJ-10 satellite time) every day and reflect heating inducing of GFP expressing.

(P) Quantification of the intensity of GFP signal in plants under the short-day ( $8 \mathrm{~h}$ light/16h dark) on oribit the SJ-10 and on ground, respectively. The GFP signals was measured as described in Materials and Methods. Values represent means for two time point images (at 15:50 and 15:55 dark period and 23:50 and 23:55 
light period every satellite day, respectively). The red asteres indicate the time points of heating shock. White areas, light; dark grey panels, dark.

Figure 4 Transcriptional responses microgravity in Arabidopsis wild-type (WT) and trangenic plants (pHSP::FT; pHSP::GFP, FG) under the Long-day (LD) and the short-day (SD) conditions, respectively.

(A) Workflow of microarray data analysis. Analysis of twofold differentially expressed genes consists of five major steps: analysis of photoperiod response genes in WT and FG plants grown on ground(1g)(step 1), identification of genes in WT and FG plants in space involved in microgravity $(\mu \mathrm{g})$ responses under LD (step 2) and SD (step 3) conditions, comparison of microgravity response genes in WT and FG plants specific to LD- or SD- conditions (Step 4) and selection of microgravity response genes in FT pathway by comparison of differetial expression genes (DEGs) in WT to those in FG (step 5).

(B) Venn diagram of transcriptomic data.

(C) Numbers of DEGs in WT and FG plants $(\mathrm{P}<0.05)$ under the LD and the SD conditions, respectively, in response to microgravity.

(D) Principal component analysis demonstrates a strong difference between the flight and the ground sample transcriptones, as well as the LD and the SD samples. Multidimensional scaling (MDS) of all DEGs in WT and FG in response to microgravity under the $\mathrm{LD}$ and the $\mathrm{SD}$, respectively.

Figure 5 Relative transcript abundance changes in WT and FG under LD- and SD-conditions in space as compared to the ground controls, respectively, were analyzed by microarray and real-time PCR. Microarray data for Cas (At5g23060), HAD (At3g48420), COL2(At3g02380) and ATM(At5g52910) are shown as an average of two independent replicates. Real-time PCR with isoform-specific primers for those genes was performed on a third independent replicates.

Figure 6 Pair-wise comparison and GO terms analysis of changes in microgravity-mediated gene expression. 
(A)Genes that are regulated in both daylength (fold changes

$\left(-1<\log _{2}(\mathrm{SD}-\mu \mathrm{g} / \mathrm{LD}-\mu \mathrm{g})<1\right.$, common) or differentially regulated under the SD versus the $\mathrm{LD}\left[\log _{2}(\mathrm{SD}-\mu \mathrm{g} / \mathrm{LD}-\mu \mathrm{g})>1\right.$ or $<-1$, daylength specific microgravity response] are depicted. The total number of genes at least twofold differentlly regulated in indicated.

(B and C) GO terms overrepresented in microgravity responses of WT (B) and FG (C) under the long-day (LD) and the short-day (SD). The significant gene ontology (PANTHER statitical overrepresentation test, GO-Slim biological precess, FDR P value<0.01) categories from up-regulated DEGs or down-regulated DEGs in WT and FG under the LD and the SD are depicted. WT, wild-type; FG, $p H S P:: F T$; $p H S P:: G F P$ trangenic plants.

Figure 7 Potential regulator of coregulated genes in protein phosphorylation funcational cluster of ' $\mu$ g-daylength-related' genes of WT and FG. (B)Four overreprented motifs enriched in upstream promoter sequences of genes in protein phosphorylation GO category in Figure $5 \mathrm{~B}$ and $\mathrm{C}$, as detected by the plant regulomics (bioinfo.sibs.ac.cn/plant-regulomics). Indicated are the P-Value representing the statistical significance of the motif. (B) Clustering analysis of the selected corregulated genes in protein phosphorylation GO category in Figure 6B and $\mathrm{C}$, which changed transcript abundance in response to microgravity in WT and FG plants under long-day (LD) and short-day (SD) conditions, respectively and have AT1G13300, KAN4, ARALYDRAFT_496250 and MNB1A binding sites in upstream promoter regions.

Figure 8 Expression of $F T$ affected photoperiod-microgravity response transcriptome. (A-C) Scatterplot showing the microgravity response of DEGs between FG and WT under the LD and the SD, respectively.

(D) Venn diagrams summarizing the number of DEGs $(\mathrm{FC}>2$ and $\mathrm{P}<0.05)$ in response to microgravity under the $\mathrm{LD}$ and the $\mathrm{SD}$, respectively, among WT and FG samples. 
(E) Selected DEGs in FG plants in response to microgravity under the LD (L- $\mu \mathrm{g})$ and the SD $(\mathrm{S}-\mu \mathrm{g})$ are compared with those in WT plants in space.

(F-H) Enriched GO Terms in DEGs of selected clusters in D. The networks graphs show BiNGO visualization of the overrepresented GO terms for the selected clusters corresponding to cluster $\mathrm{C} 1$ to $\mathrm{C} 3$ indicating in $\mathrm{E}$, respectively. Categories in GoslimPlants(Breeze et al., 2011) were used to simplify this analysis and the same nodes are shown on all three graphs. Uncolored nodes are not overreprensented, but they may be the parents of overrepresented terms. Colored nodes represent GO terms that are significantly overrepresented (Benjamini and Hochberg corrected P value $<0.05$ ), with the shade indicating significance as shown in the color bar.

Figure 9 The core photoperiod response genes altered expression levels by expouse to microgravity.

(A) $\log _{2}$ FC of the 20 core photoperiod genes in the FG under LD or SD on ground $(1 \mathrm{~g})$ and microgravity in space $(\mu \mathrm{g})$, respectively, in comparison with these genes in wild-type (WT) under the same condition (FG/WT).

(B) Diagram of the protein interaction networks of the photoperiod response genes. The genes, which altered expression level in response to microgravity in space in comparison with their controls on ground, were labelled by colour in yellow and $\log _{2} \mathrm{FC}$ of these highlighted genes are indicated in $\mathrm{C}$.

(C) $\log _{2} \mathrm{FC}$ of selected core photoperiod genes in WT and FG in response to microgravity under the LD and the SD condition, respectively.

\section{Supplementary data}

Supplementary Figure S1 Heat shock activation of GFP gene expresion in pHSP::FG, $p H S P:: G F P(\mathrm{FG})$ transigenic plants.

(A and E) 14-day-old wild-type (A) and FG transgenic seedlings (E) were treated by $37^{\circ} \mathrm{C}$ for $1 \mathrm{~h}$ or under $20^{\circ} \mathrm{C}$ control conditions, respectively.

(B-D) Fluorescence images of leaves from $37^{\circ} \mathrm{C}$ heat treated seedlings and $20^{\circ} \mathrm{C}$ control plants, respectively. 
(F-H) image of leaves under differential interence contrast optics microscope.

Supplementary Figure S2 Heat shock treatment induced FT expression and flowering. All plants were 20 -day old and were subjected to heat shock $\left(37^{\circ} \mathrm{C}\right)$ induction for $2 \mathrm{~h}$ at day 15 after germination.

(A, D) Phonetype of wild-type (WT) plants were grown under the long-day (16 h light/8h dark, LD) and short-day (8 h light/16h dark, SD) conditions, respectively .

$(\mathrm{B}, \mathrm{E})$ Phonetype of transgenic plants $p H S P:: G F P$ were grown under the LD and the SD conditions, respectively.

(C, F) Phonetype of transgenic plants $p H S P:: F T, p H S P:: G F P$ were grown under the LD and the SD conditions, respectively.

(G) qRT-PCR analysis of $F T$ transcript levels in 16-day-old $p H S P:: F T, p H S P:: G F P$ plants grown under the SD conditions with or without the $2 \mathrm{~h}$ heat shock treatment $\left(37^{\circ} \mathrm{C}\right)$.

Note that the plants $p H S P:: F T$,pHSP ::GFP under both LD- and SD-conditions (C and F) appeared early flowering in comparison with their controls of WT(A and D) and $p H S P:: G F P$ (B and $\mathrm{E}$ ) under the same conditions.

Supplementary Table S1 Expression data of identified genes of wild-type (WT) plants grown under the short-day (SD) on ground was compared with those under the long-day (LD) on ground(WT- $1 \mathrm{~g}-\mathrm{SD}$ versus WT-1g-LD).

Supplementary Table S2 Expression data of identified genes of $p H S P: \because F T$, pHSP:GFP (FG) plants grown under the short-day (SD) on ground was compared with those under the long-day (LD) on ground(FG- 1g-SD versus FG-1g-LD). Supplementary Table S3 Expression data of identified genes of wild-type (WT) plants grown under the long-day (LD) in microgravity $(\mu \mathrm{g})$ in space were compared with those under the LD on ground(WT- $\mu$ g-LD versus WT-1g-LD).

Supplementary Table S4 Expression data of identified genes of $p H S P:: F T$, $p H S P: G F P(\mathrm{FG})$ transgenic plants grown under the long-day (LD) in microgravity $(\mu \mathrm{g})$ in space were compared with those under the LD on ground(FG- $\mu \mathrm{g}-\mathrm{LD}$ versus FG-1g-LD). 
Supplementary Table S5 Expression data of identified genes of wild-type (WT) plants grown under the short-day $(\mathrm{SD})$ in microgravity $(\mu \mathrm{g})$ in space were compared with those under the SD on ground(WT- $\mu$ g-SD versus WT-1g-SD).

Supplementary Table S6 Expression data of identified genes of $p H S P:: F T$, pHSP:GFP (FG) transgenic plants grown under the short-day (SD) in microgravity $(\mu \mathrm{g})$ in space were compared with those under the SD on ground(FG- $\mu$ g-SD versus FG-1g-SD).

Supplementary Table S7 Pair-wise comparison of altered expression of genes in response to microgravity in WT under the SD with those under the LD conditions. Supplementary Table S8 Pair-wise comparison of altered expression of genes in response to microgravity in $p H S P:: F T$, $p H S P: G F P(\mathrm{FG})$ transgenic plants under the SD with those under the LD conditions.

Supplementary Table S9 Data for the selected clusters corresponding to cluster C1 to $\mathrm{C} 3$ indicating in Figure 7E.

Supplementary Table S10 Protein phosphorylation proteins encoded by day-length related microgravity-responsive genes.

Supplementary Table S11 Microgravity response of core photoperiod response genes in WT and/or FG plants grown in space. Genes identified with a significant $(\mathrm{FC}>2$ and $\mathrm{p}<0.05)$ change in expersion level. 


\section{Figures}
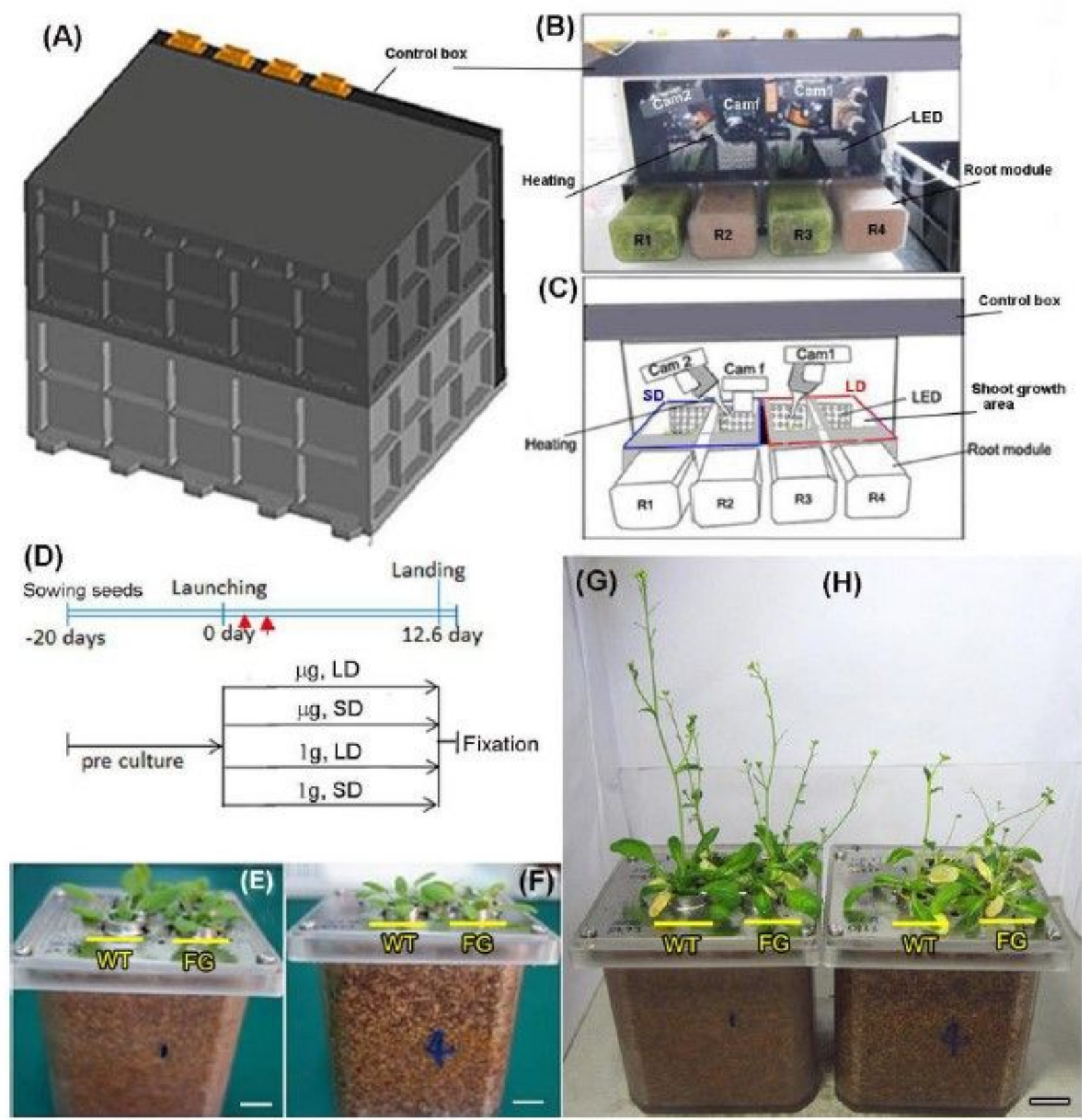

\section{Figure 1}

Experimental setup on board the Chinese recoverable satellite SJ-10. (A) The external view of the plant growth unit $(P G U)(H \times W \times D=370 \times 270 \times 270 \mathrm{~mm})$ with the cover. (B) The inside view of the PGU without the cover, as described in (C). (C) Diagram of PGU used on board the Chinese satellite SJ-10. Showing the distribution and state of components and samples in PGU during space flight. The components included four root modules, three cameras, two fans and heating parts, and four LED banks with controlled longday (LD) or short-day (SD) photoperiods, respectively. indicating LED plates; heating systems; video CCD cameras(Cam 1 and Cam 2); the GFP imaging camera (Cam f) and root modules (R1 and R3 with rice seedlings; R2 and R4 with Arabidopsis seedlings). (D)An overview of the time process of the space 
experiment. wild-type and transgenic Arabidopsis plants were germinated and grown in the root module in green house on ground for $20 \mathrm{~d}$ after sowing. At this age, the plants had formed four rosette leaves, when they were loaded into PGU less than 24h prior to take off. Heating treatment (red arrows point) to activated expression of pHSP::FT; pHSP::GFP was performed at day 2 on orbiter. The plants in PGU were grown under long-day (LD, $16 \mathrm{~h}$ light/8 h dark) and short-day(SD, $8 \mathrm{~h}$ light/16 h dark) conditions in space $(\mu \mathrm{g})$ and on ground $(1 \mathrm{~g})$, respectively. (E and F) 20-day old wild-type (WT) and transgenic (pHSP::FT; pHSP::GFP, FG) Arabidopsis seedlings grown in the root modules before being loaded into the PGU. (G and $H$ ) The situation of samples recovered from spaceflight under $L D(G)$ and SD $(H)$ conditions. Bars $=1 \mathrm{~cm}$. 

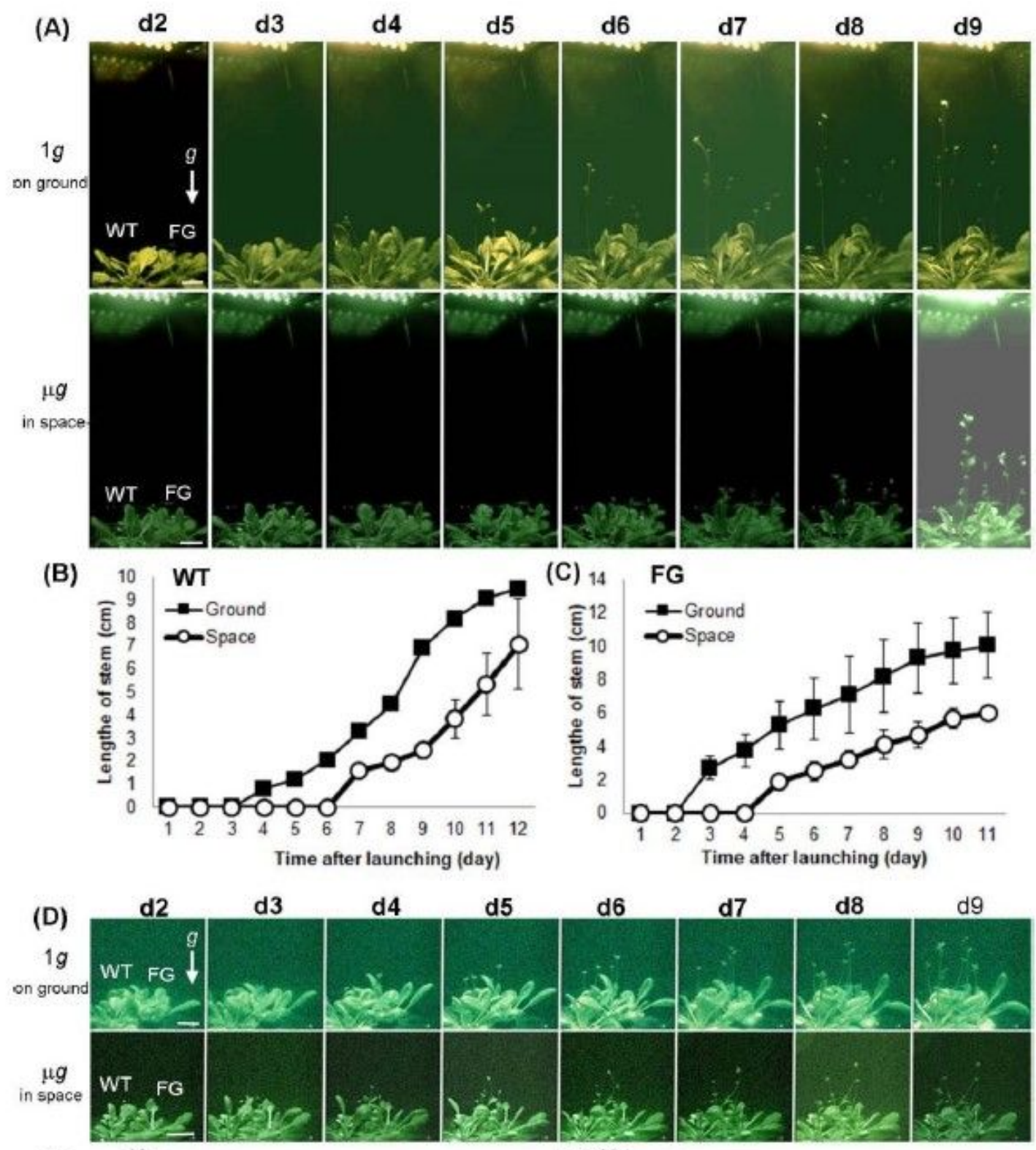

(E)

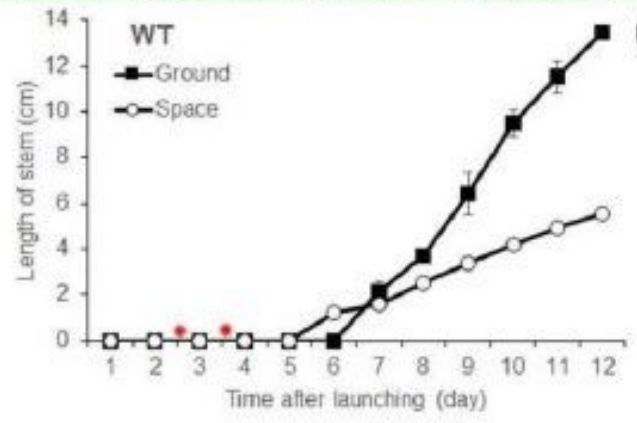

(C) $14, \quad$ FG
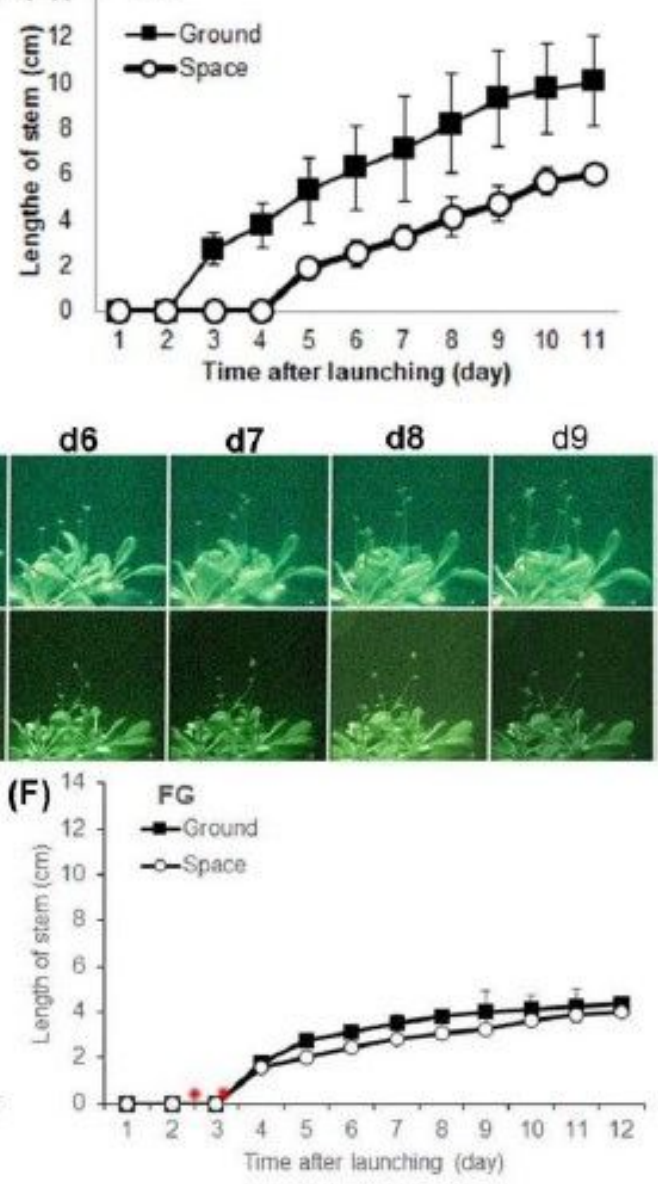

\section{Figure 2}

Floral transition time of wild-type and pHSP::FT; pHSP::GFP transgenic plants grown under the long-day condition on ground and in flight on board the SJ-10 satellite. (A) example images of plants grown under long-day condition on ground( $1 \mathrm{~g}$, on ground) and in space ( $\mu \mathrm{g}$, in space) at day 2 (d2) to day 9 (d9) after satellite took off. (B and C) comparison of floral transition time and average length of stems of wild-type (WT) and pHSP::FT; pHSP::GFP (FG) transgenic plants under the long-day (LD) in space with their 
controls on ground. Data are determined from the living images of plants on orbit in space or on ground. $\mathrm{n}=4$ plants per treatment. (D) example images of plants of wild-type and transgenic plants under the short-day (SD) condition on ground and in space at $d 2$ to $d 9$ after satellite took off. (E and F) comparison of floral transition time and average length of stems of WT and FG plants treated by heating shock (red asteres indicate) under the SD condition in space and on ground. Data are determined from the living images downloaded from the plant growth units in space and on ground. $n=4$ plants per treatment. Bars in $(A)$ and $(D)=10 \mathrm{~mm}$.

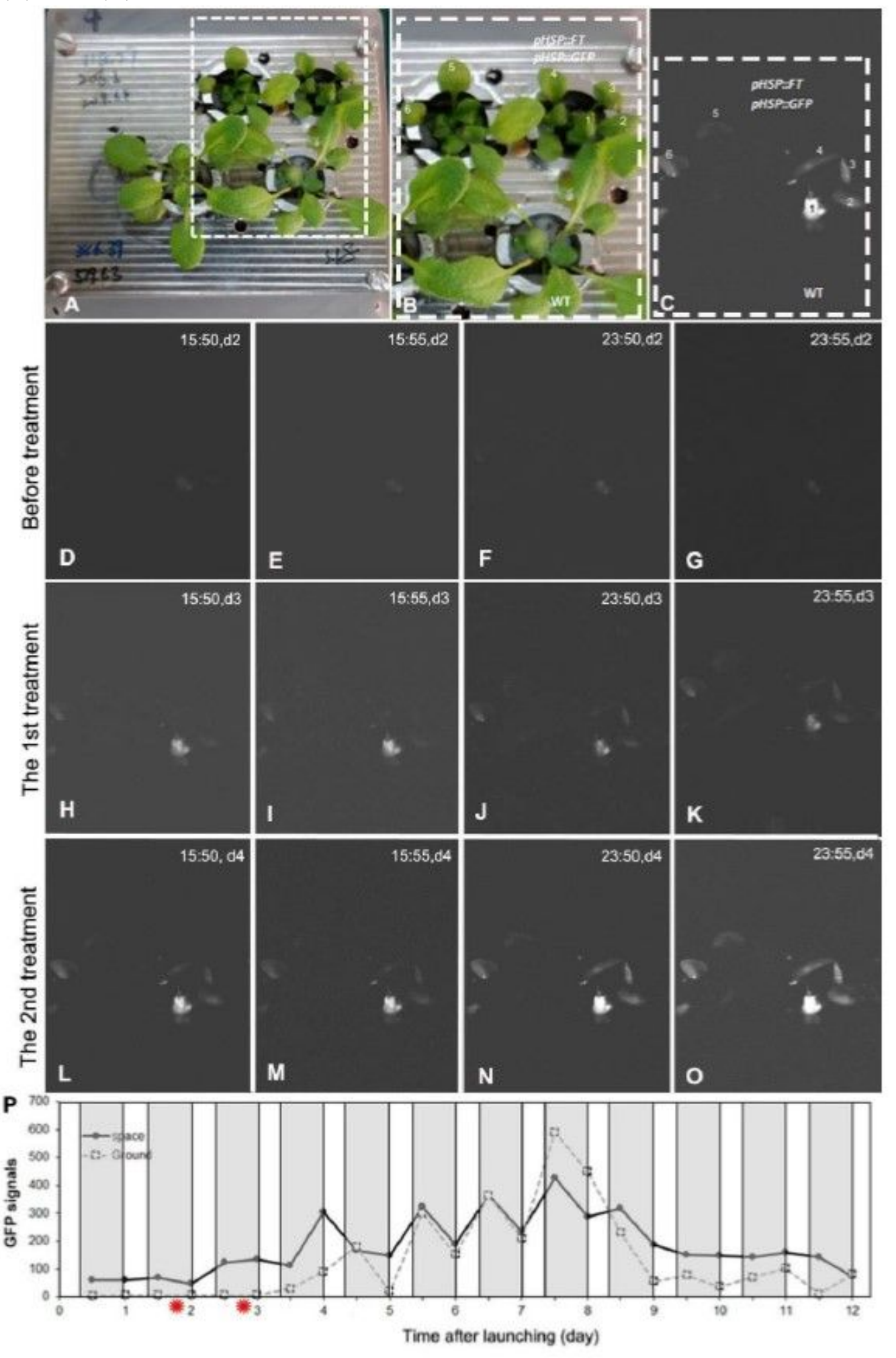




\section{Figure 3}

GFP expressing in short-day grown plants treated by $37^{\circ} \mathrm{C}$ heating on board SJ-10. (A) pHSP:::FT; pHSP::GFP transgenic plants and wild-type (WT) plants photographed in white light before launching. The framed region in (A) is detailed in (B). (C) a represent GFP image showed the expression of GFP of leaves1-6 in (B) (D-0) Example GFP images were captured by the GFP imager at day 2 to day 4 after heating treatment. The images were captured at four time points $(15: 50,15: 55,23: 50$ and 23:55, on board SJ-10 satellite time) every day and reflect heating inducing of GFP expressing. (P) Quantification of the intensity of GFP signal in plants under the short-day (8h light/16h dark) on oribit the SJ-10 and on ground, respectively. The GFP signals was measured as described in Materials and Methods. Values represent means for two time point images (at 15:50 and 15:55 dark period and 23:50 and 23:55 light period every satellite day, respectively). The red asteres indicate the time points of heating shock. White areas, light; dark grey panels, dark. 
(A)

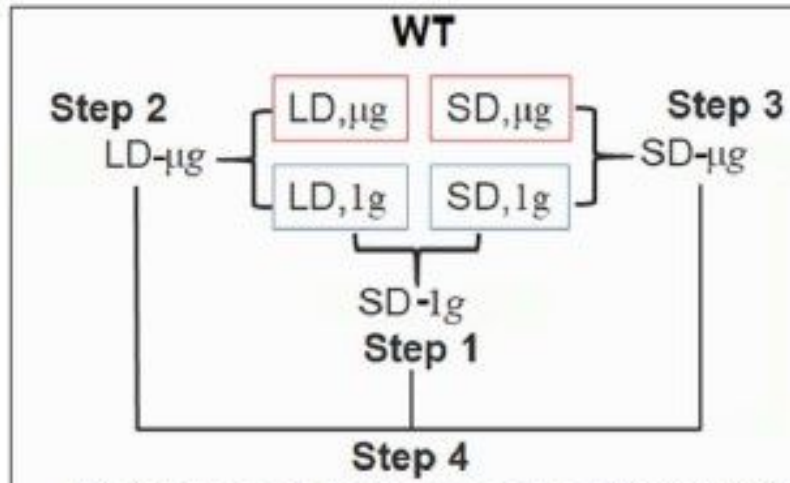

Photoperiod related $\mu \mathrm{g}$ response genes in WT

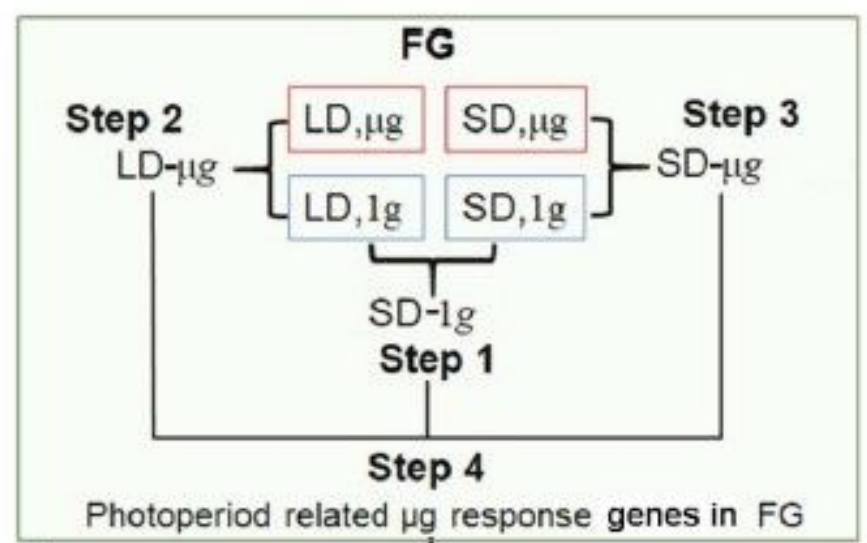

Photoperiod related $\mu \mathrm{g}$ response genes in FG

\section{Step 5}

FT regulated $\mu \mathrm{g}$ response
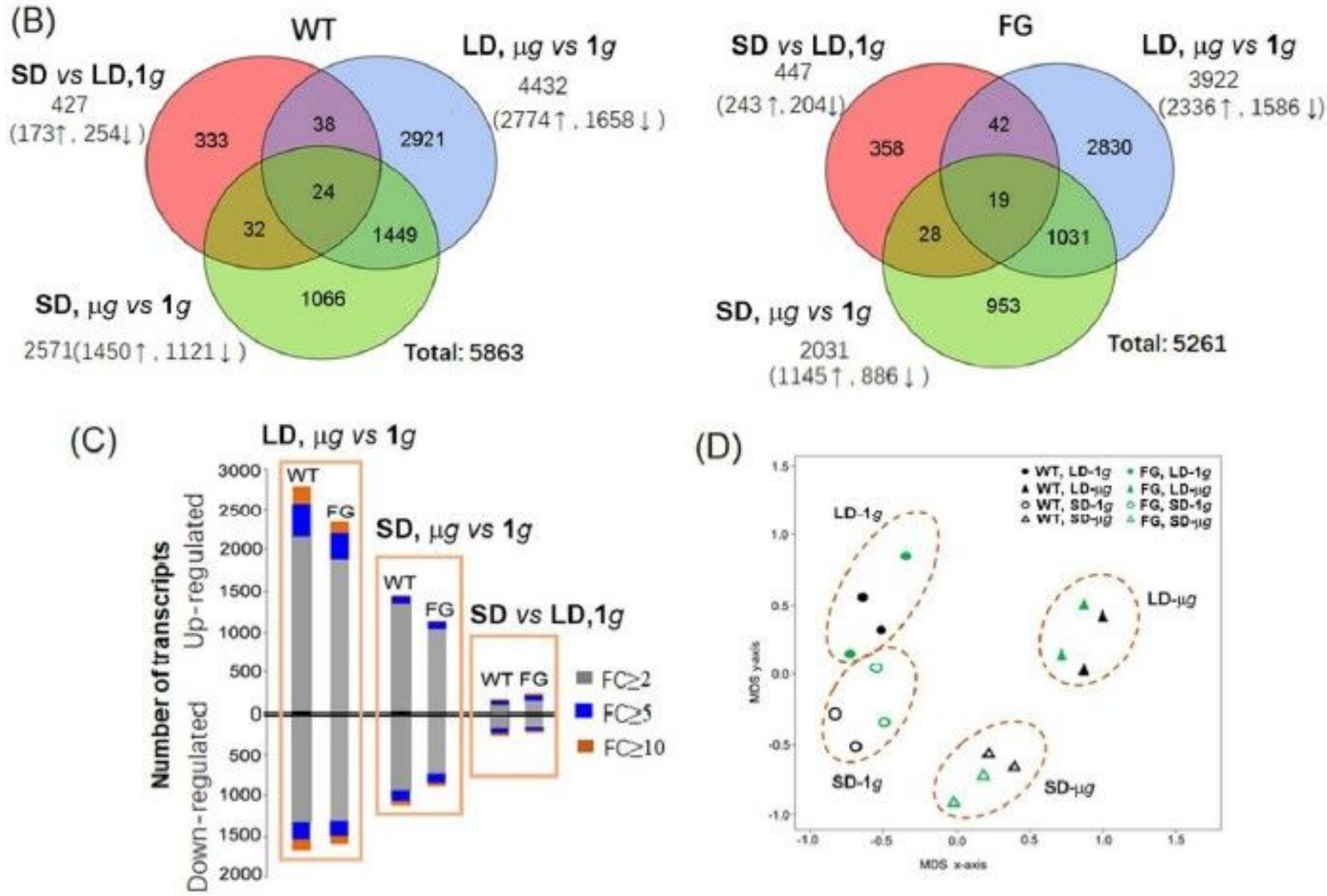

\section{Figure 4}

Transcriptional responses microgravity in Arabidopsis wild-type (WT) and trangenic plants (pHSP::FT; pHSP::GFP, FG) under the Long-day (LD) and the short-day (SD) conditions, respectively. (A) Workflow of microarray data analysis. Analysis of twofold differentially expressed genes consists of five major steps: analysis of photoperiod response genes in WT and FG plants grown on ground(1g)(step 1), identification of genes in WT and FG plants in space involved in microgravity $(\mu \mathrm{g})$ responses under LD (step 2$)$ and SD 
(step 3) conditions, comparison of microgravity response genes in WT and FG plants specific to LD- or SD- conditions (Step 4) and selection of microgravity response genes in FT pathway by comparison of differetial expression genes (DEGs) in WT to those in FG (step 5). (B) Venn diagram of transcriptomic data. (C) Numbers of DEGs in WT and FG plants $(P<0.05)$ under the LD and the SD conditions, respectively, in response to microgravity. (D) Principal component analysis demonstrates a strong difference between the flight and the ground sample transcriptones, as well as the LD and the SD samples. Multidimensional scaling (MDS) of all DEGs in WT and FG in response to microgravity under the LD and the SD, respectively.

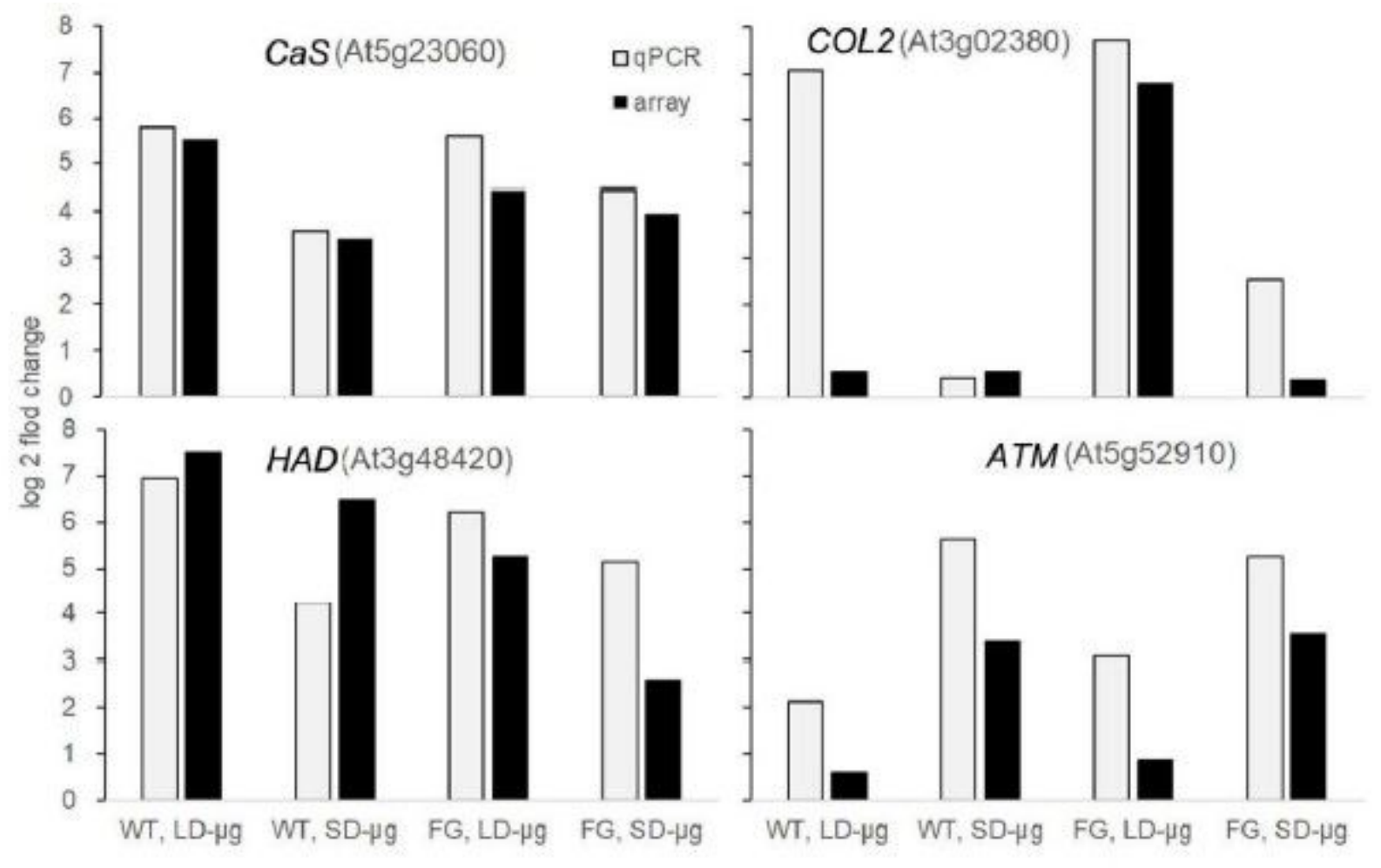

Figure 5

Relative transcript abundance changes in WT and FG under LD- and SD-conditions in space as compared to the ground controls, respectively, were analyzed by microarray and real-time PCR. Microarray data for Cas (At5g23060), HAD (At3g48420), COL2(At3g02380) and ATM(At5g52910) are shown as an average of two independent replicates. Real-time PCR with isoform-specific primers for those genes was performed on a third independent replicates. 


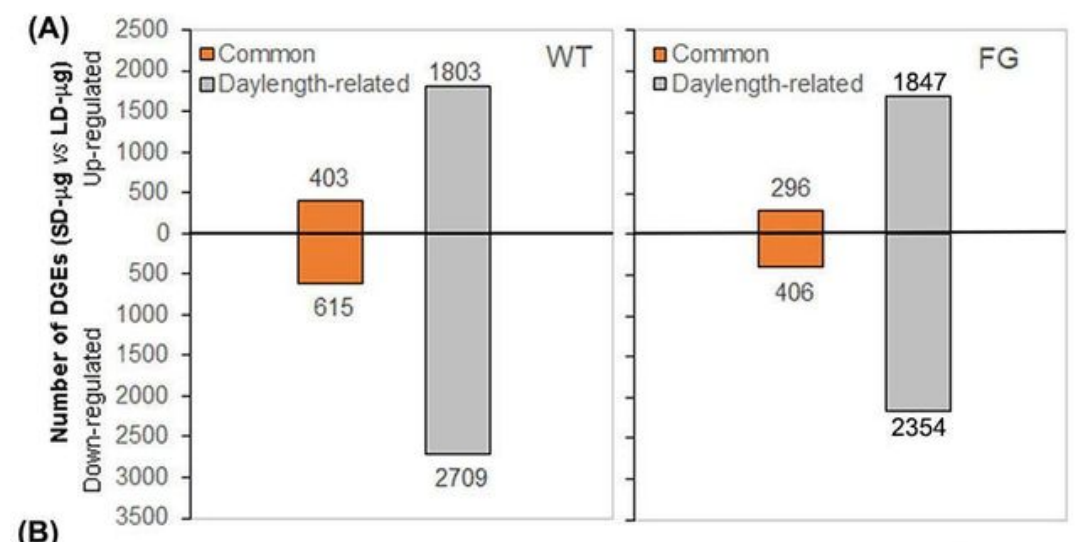

(B)

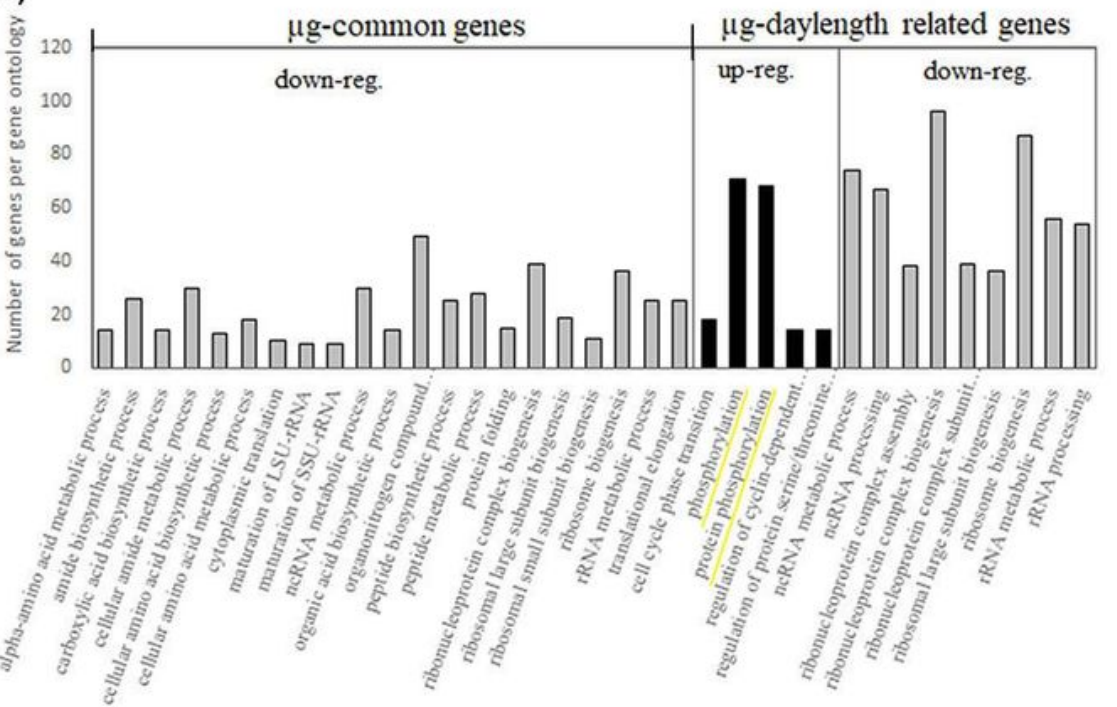

(C)

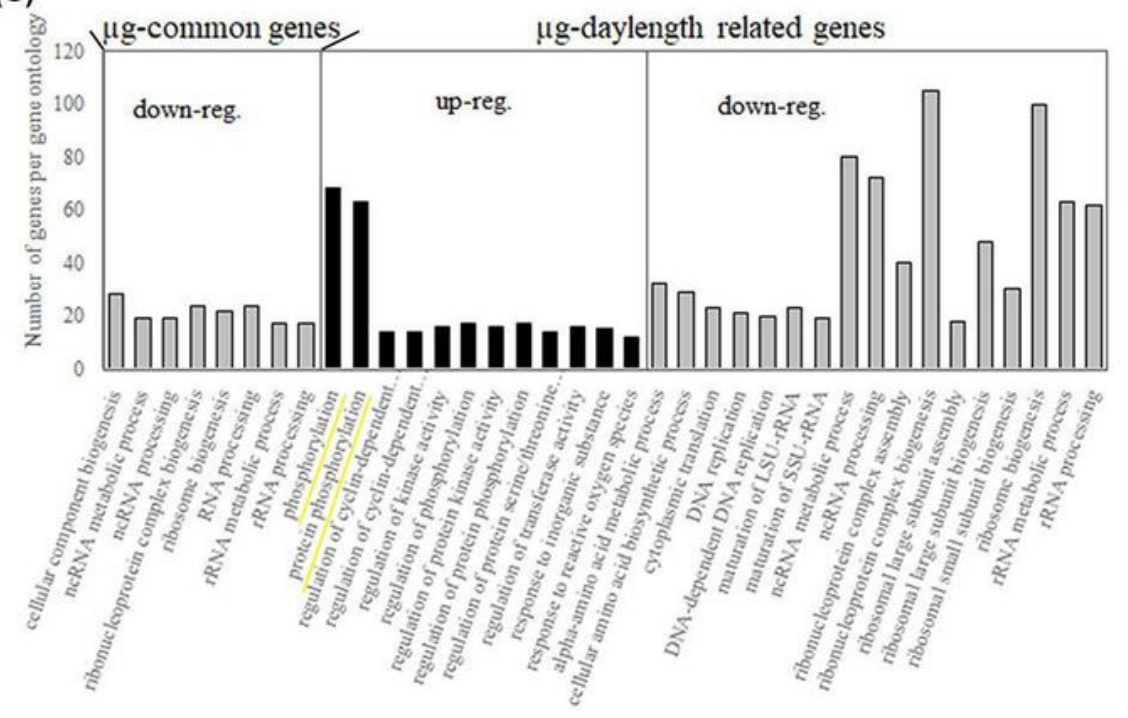

Figure 6

Pair-wise comparison and GO terms analysis of changes in microgravity-mediated gene expression. (A)Genes that are regulated in both daylength (fold changes $(-1<\log 2(S D-\mu g / L D-\mu g)<1$, common) or differentially regulated under the SD versus the LD [log2(SD- $\mu \mathrm{g} / \mathrm{LD}-\mu \mathrm{g})>1$ or $<-1$, daylength specific microgravity response] are depicted. The total number of genes at least twofold differentlly regulated in indicated. (B and C) GO terms overrepresented in microgravity responses of WT (B) and FG (C) under the 
long-day (LD) and the short-day (SD). The significant gene ontology (PANTHER statitical overrepresentation test, GO-Slim biological precess, FDR P value $<0.01$ ) categories from up-regulated DEGs or down-regulated DEGs in WT and FG under the LD and the SD are depicted. WT, wild-type; FG, pHSP::FT; pHSP::GFP trangenic plants.

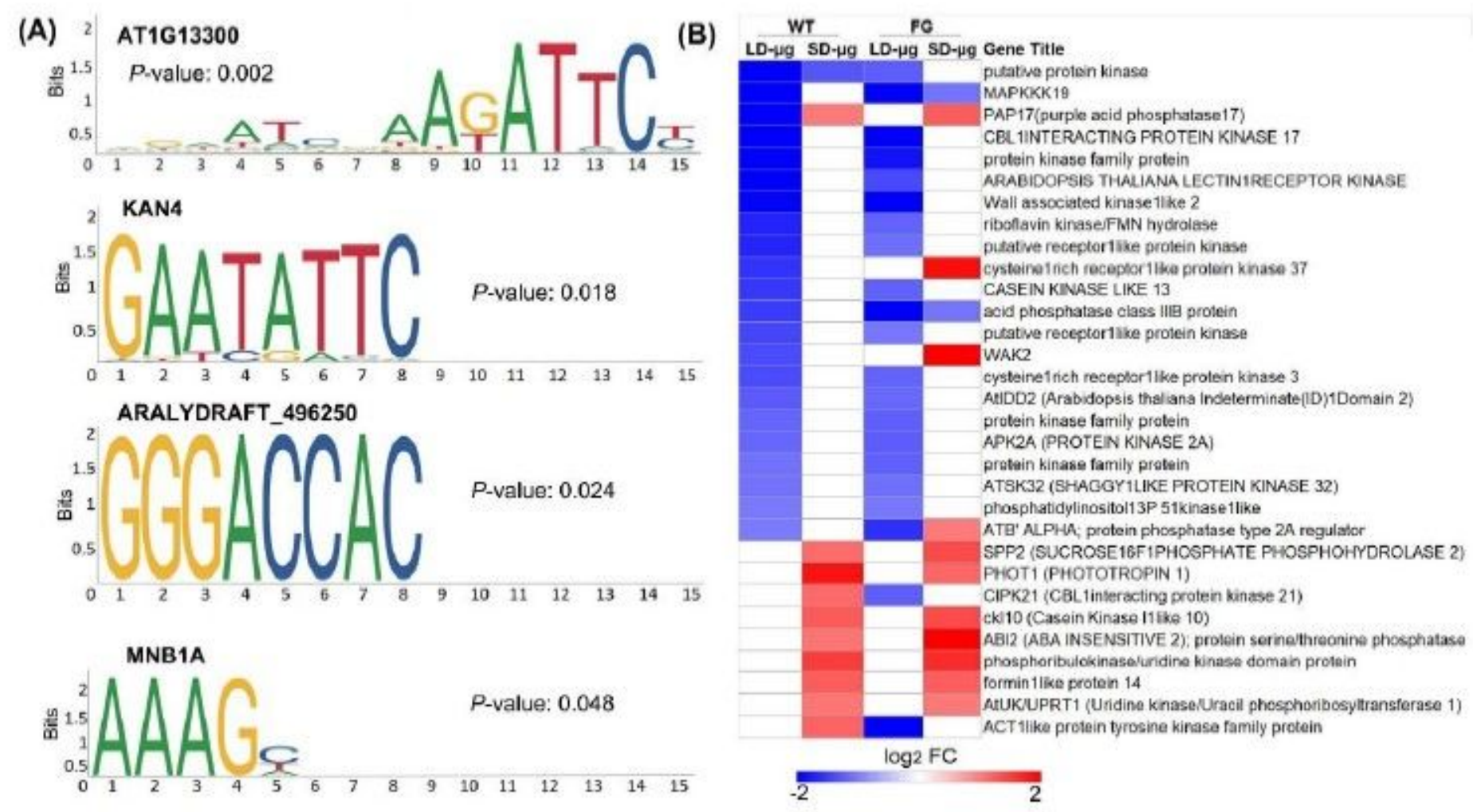

\section{Figure 7}

Potential regulator of coregulated genes in protein phosphorylation funcational cluster of ' $\mu$ g-daylengthrelated' genes of WT and FG. (B)Four overreprented motifs enriched in upstream promoter sequences of genes in protein phosphorylation $\mathrm{GO}$ category in Figure $5 \mathrm{~B}$ and $\mathrm{C}$, as detected by the plant regulomics (bioinfo.sibs.ac.cn/plant-regulomics). Indicated are the P-Value representing the statistical significance of the motif. (B) Clustering analysis of the selected corregulated genes in protein phosphorylation $\mathrm{GO}$ category in Figure 6B and $\mathrm{C}$, which changed transcript abundance in response to microgravity in WT and FG plants under long-day (LD) and short-day (SD) conditions, respectively and have AT1G13300, KAN4, ARALYDRAFT_496250 and MNB1A binding sites in upstream promoter regions. 

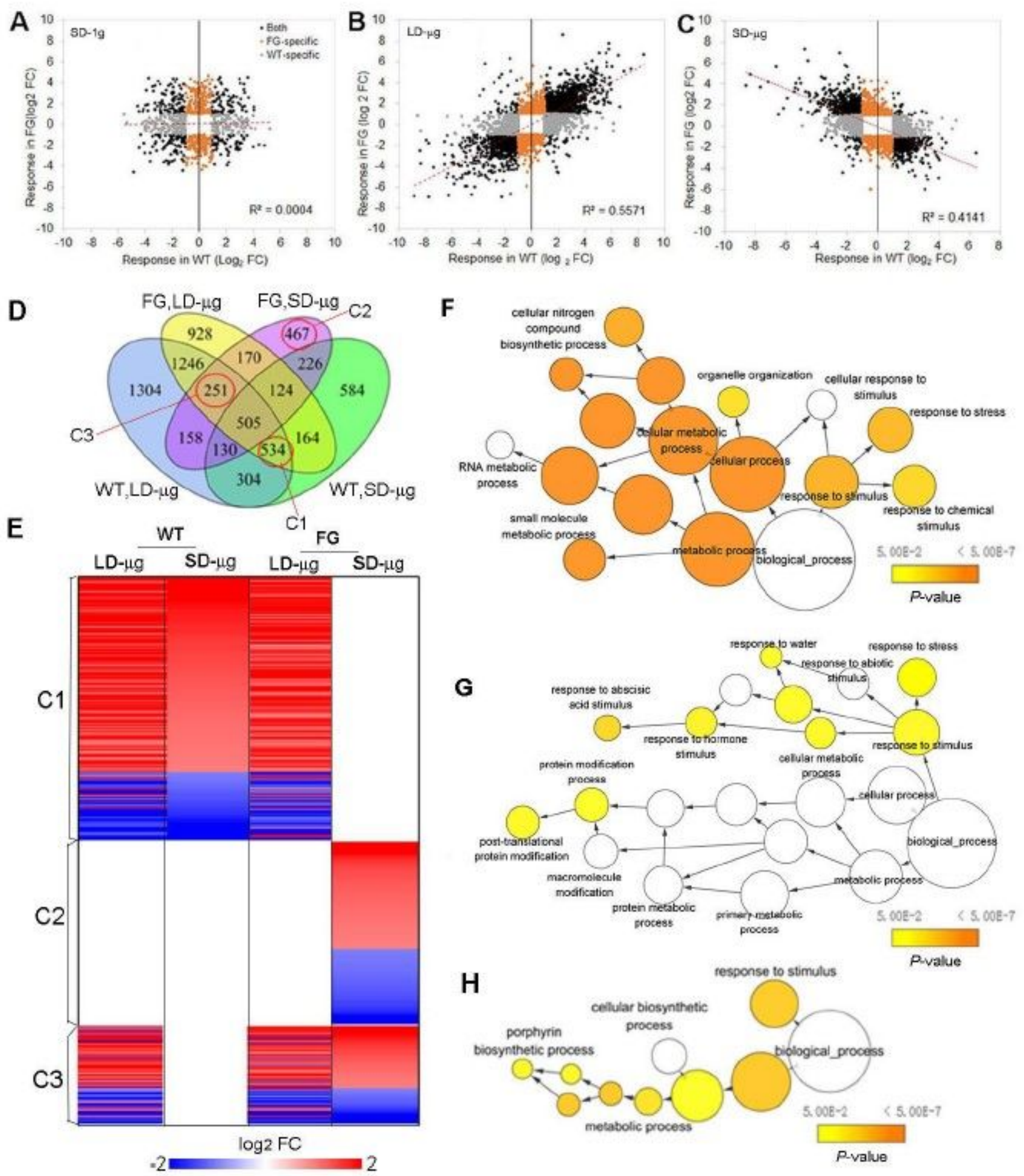

\section{Figure 8}

Expression of FT affected photoperiod-microgravity response transcriptome. (A-C) Scatterplot showing the microgravity response of DEGs between FG and WT under the LD and the SD, respectively. (D) Venn diagrams summarizing the number of DEGs $(F C>2$ and $P<0.05)$ in response to microgravity under the $L D$ and the SD, respectively, among WT and FG samples. (E) Selected DEGs in FG plants in response to microgravity under the LD $(\mathrm{L}-\mu \mathrm{g})$ and the SD $(\mathrm{S}-\mu \mathrm{g})$ are compared with those in WT plants in space. (F-H) 
Enriched GO Terms in DEGs of selected clusters in D. The networks graphs show BiNGO visualization of the overrepresented $\mathrm{GO}$ terms for the selected clusters corresponding to cluster $\mathrm{C} 1$ to $\mathrm{C} 3$ indicating in $\mathrm{E}$, respectively. Categories in GoslimPlants(Breeze et al., 2011) were used to simplify this analysis and the same nodes are shown on all three graphs. Uncolored nodes are not overreprensented, but they may be the parents of overrepresented terms. Colored nodes represent $\mathrm{GO}$ terms that are significantly overrepresented (Benjamini and Hochberg corrected $P$ value $<0.05$ ), with the shade indicating significance as shown in the color bar.
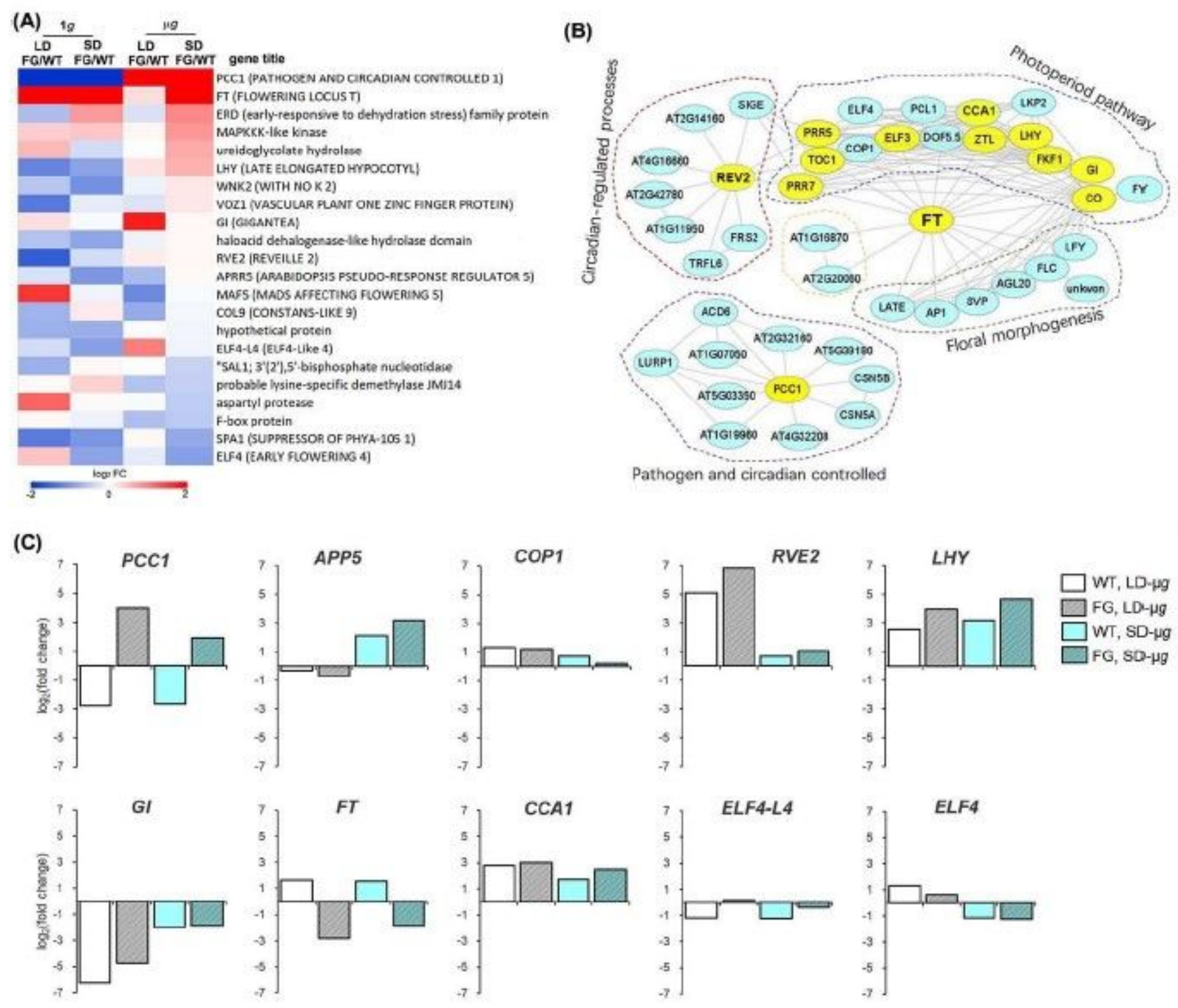

\section{Figure 9}

The core photoperiod response genes altered expression levels by expouse to microgravity. (A) Log2 FC of the 20 core photoperiod genes in the FG under LD or SD on ground $(1 \mathrm{~g})$ and microgravity in space $(\mu \mathrm{g})$, respectively, in comparison with these genes in wild-type (WT) under the same condition (FG/WT). (B) Diagram of the protein interaction networks of the photoperiod response genes. The genes, which altered expression level in response to microgravity in space in comparison with their controls on ground, were 
labelled by colour in yellow and log2 FC of these highlighted genes are indicated in C. (C) Log2 FC of selected core photoperiod genes in WT and FG in response to microgravity under the LD and the SD condition, respectively.

\section{Supplementary Files}

This is a list of supplementary files associated with this preprint. Click to download.

- SuppITabS1SD1ginWT.xIsx

- SupplTabS2SD1ginFG.xlsx

- SupplTabS3LDuginWT.xlsx

- SuppITabS4LDuginFG.xIsx

- SupplTabS5SDuginWT.xlsx

- SupplTabS6SDuginFG.xlsx

- SuppITabS7LDvsSDuginWT.xIsx

- SuppITabS8LDvsSDuginFG.xIsx

- SuppITabS9.xlsx

- SupplTabS10.xIsx

- SupplTabS11.xlsx

- Supplfigures20210407.pdf 